\title{
Water Impact Test and Simulation of a Composite Energy Absorbing Fuselage Section
}

\author{
Edwin L. Fasanella and Karen E. Jackson \\ e.l.fasanella@larc.nasa.gov, k.e.Jackson@larc.nasa.gov \\ US Army Research Laboratory, Vehicle Technology Directorate \\ Hampton, VA \\ Chad Sparks and Ashish Sareen \\ CSparks@bellhelicopter.textron.com, ASareen@bellhelicopter.textron.com \\ Bell Helicopter Textron, Inc. \\ Fort Worth, TX
}

\begin{abstract}
In March 2002, a 25-ft/s vertical drop test of a composite fuselage section was conducted onto water. The purpose of the test was to obtain experimental data characterizing the structural response of the fuselage section during water impact for comparison with two previous drop tests that were performed onto a rigid surface and soft soil. For the drop test, the fuselage section was configured with ten $100-\mathrm{lb}$. lead masses, five per side, that were attached to seat rails mounted to the floor. The fuselage section was raised to a height of $10-\mathrm{ft}$. and dropped vertically into a $15-\mathrm{ft}$. diameter pool filled to a depth of 3.5-ft. with water. Approximately 70 channels of data were collected during the drop test at a $10-\mathrm{kHz}$ sampling rate. The test data were used to validate crash simulations of the water impact that were developed using the nonlinear, explicit transient dynamic codes, MSC.Dytran and LS-DYNA. The fuselage structure was modeled using shell and solid elements with a Lagrangian mesh, and the water was modeled with both Eulerian and Lagrangian techniques. The fluid-structure interactions were executed using the "fast" general coupling in MSC.Dytran and the Arbitrary Lagrange-Euler (ALE) coupling in LS-DYNA. Additionally, the smooth particle hydrodynamics (SPH) meshless Lagrangian technique was used in LS-DYNA to represent the fluid. The simulation results were correlated with the test data to validate the modeling approach. Additional simulation studies were performed to determine how changes in mesh density, mesh uniformity, fluid viscosity, and failure strain influence the test-analysis correlation.
\end{abstract}

\section{Introduction}

Considerable research has been performed on test and analysis of impacts into water and soft-soil [1-3]. However, the research has raised questions regarding the severity of the impacts, whether the onset rate of the acceleration pulse is greater in water than onto a hard surface, and whether an airframe designed for hard surface impact behaves differently for soft-soil and water impact. Thus, the focus of this project is to examine these questions, and to determine whether nonlinear dynamic finite element analyses can simulate the water impact with good fidelity. In 2001, a cooperative agreement was established between Bell Helicopter, the National Rotorcraft Technology Center/Rotorcraft Industry Technology Association (NRTC/RITA) and the US Army Research Lab, Vehicle Technology Directorate (ARLVTD) to investigate the crashworthy response of a composite fuselage section for multi-terrain impact. Under this agreement, impact tests of the composite fuselage section shown schematically in Figure 1 were performed for nearly identical configurations onto a rigid surface [4], soft soil [1], and water.

\footnotetext{
Presented at the American Helicopter Society 59 th Annual Forum, Phoenix, AZ, May 6-8, 2003. This paper is a work of the US Government and is, therefore, in the public domain.
}

In conjunction with the testing program, crash simulations for each impact surface were performed and the models were validated through correlation of the analytical and experimental data $[1,4]$. This paper summarizes the experimental data obtained during the water impact test, describes the modeling approach and execution, and presents the correlation between the test data and the analytical predictions.

\section{Description of the Fuselage Section}

A research program was conducted at NASA Langley Research Center to develop a composite fuselage concept, illustrated in Figure 1, for improved crashworthiness [5-13]. In 1999, the first full-scale prototype was fabricated and tested. Since that time several additional impact tests of the composite fuselage section have been performed including a test to evaluate its crashworthy performance with seats and dummies [13]. The composite fuselage section is approximately 5 -ft. long and 5 - $\mathrm{ft}$. in diameter. The stiff structural floor produces a uniform global crushing of the energy-absorbing subfloor, which consists of five uniformly spaced blocks of crushable Rohacell 31-IG closed-cell foam overlaid with E-glass/epoxy facesheets. The cross-sectional geometry of the Rohacell foam blocks, shown in Figure 1, was designed to achieve a fairly uniform crushing stress. The floor loading was provided by ten $100-\mathrm{lb}$. lead weights that were mounted to the floor five per side through the seat tracks. 


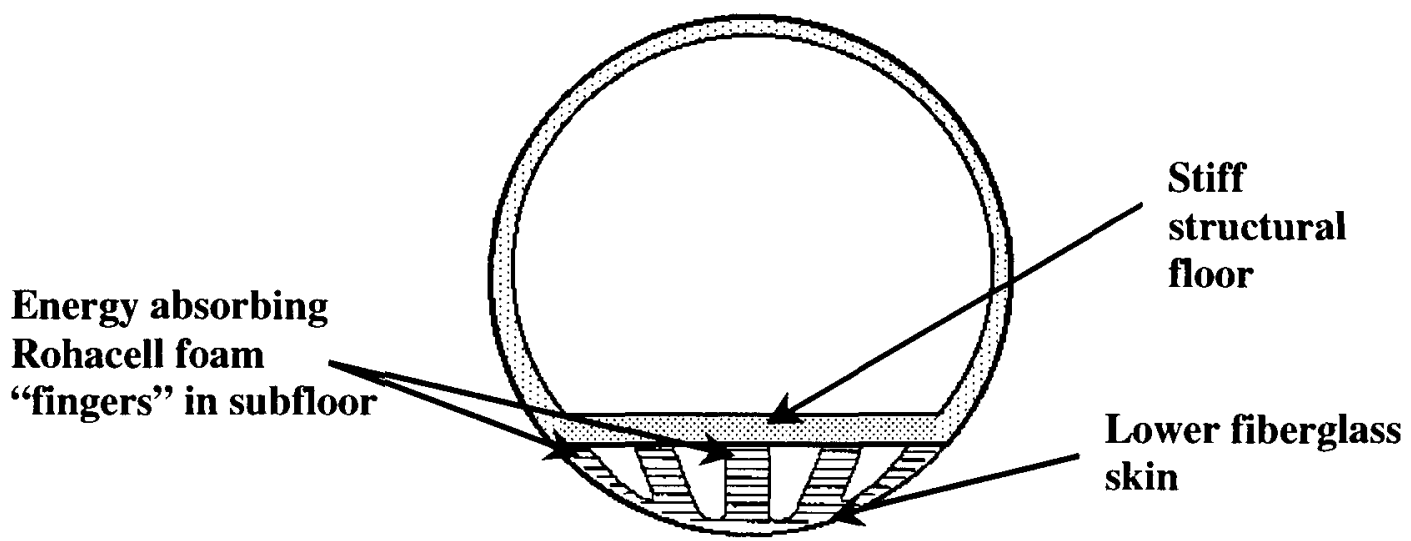

Figure. 1. Front schematic drawing of the composite fuselage section.

Because the subfloor consists of five discreet Rohacell foam blocks, the lower fiberglass skin is unsupported between the foam blocks. This design is somewhat analogous to a conventional metallic ring-frame and skin structure. For such structures, the skin between the blocks (or frames) is highly loaded upon impact onto nonrigid surfaces. The energy absorbing features of the composite fuselage section were designed for a rigid surface impact, and the fact that the skin is unsupported between the foam blocks makes this an interesting test article for soft-soil and water impacts.

\section{Experimental Program}

In March 2002, a vertical drop test of the composite fuselage section was conducted into a $3.5-\mathrm{ft}$. deep, $15-\mathrm{ft}$. diameter pool of water. The empty fuselage section weighed 208 pounds. The test article consisting of the fuselage section outfitted with instrumentation, seat rails, and ten 100-lb. lead masses attached to the floor weighed 1,226 pounds. The test was performed using the $70-\mathrm{ft}$. drop tower located at the Impact Dynamics Research Facility at NASA Langley Research Center. The fuselage section was raised to a height of 10 feet above the water and dropped into the pool to achieve a $25.4-\mathrm{ft} / \mathrm{s}$ vertical velocity at impact. The section impacted with a forwarddown pitch of approximately 1 degree and a roll of 0 degree.

To facilitate comparisons of the test data, the fuselage section was instrumented with 67 accelerometers located at the same positions as for the section that was dropped onto concrete in 2000 and the section that was dropped onto sand in 2001. A schematic drawing illustrating the instrumentation layout on the floor is shown in Figure 2. Note that selected accelerometer positions are numbered in Figure 2, corresponding to the original channel numbers. All accelerometers on the floor were oriented vertically. Inboard and outboard accelerometers were located on the bolts securing the large lead masses to the aluminum seat rails. The accelerometers positioned between the seat rails were mounted on blocks adhered directly to the floor. Channels 1, 10,42, and 33 are located at the front of the section. From symmetry, one would expect that data from inboard channels 10,42,18, and 50; and from outboard channels $1,33,9$, and 41 would look very similar for a flat impact. However, since the front end was pitched down by 1 degree, front to back symmetry was violated. The data were collected with a digital data acquisition system (DAS), located external to the fuselage section, at a $10-\mathrm{kHz}$ sampling rate.

To measure the pressure pulse, seven pressure transducers were secured into the Rohacell foam blocks on the fuselage bottom surface. Three of the pressure transducers were placed in the center foam block, with one at the bottom center and the other two in the gaps about 4 inches to the right and left of the center-line as illustrated in Figure 3. The remaining four transducers were placed at the bottom center of the other four blocks. Once the outer fiberglass skin was applied over the five foam blocks, small circular holes were cut into the skin to allow the face of each pressure transducer to contact the water during the impact. A photograph made after the transducers and wiring were installed, but before the fiberglass skin was applied is shown in Figure 4. The pressure data were collected at $10-\mathrm{kHz}$ sampling rate using the digital DAS.

Prior to the drop test, all connectors were wrapped with plastic material and taped. To further protect the instrumentation, cables, and connectors from water intrusion; the entire floor area of the section was "vacuum-bagged" as shown in Figure 5. Special attention was given to sealing the instrumentation wiring. After a good vacuum was drawn, the vacuum hose was sealed. Although air leakage occurred after sealing, it was postulated that any trapped air inside the "vacuum bag" would exert a positive differential pressure within the bag that should keep water from entering. This behavior was observed after the test as air pockets did form within the bag. However, no water was observed to penetrate the sealed area after the vacuum bag was removed post-test. 


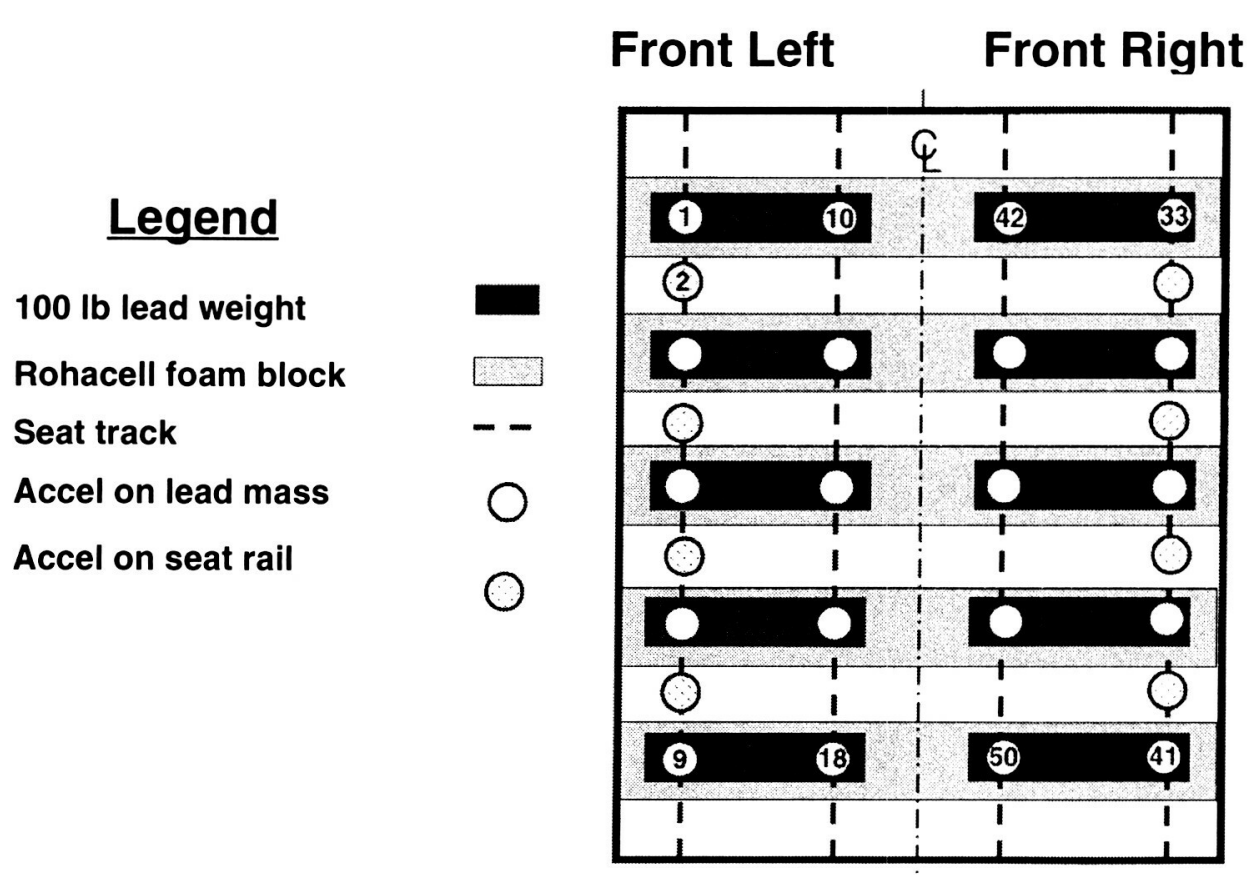

Figure. 2. Floor diagram showing placement of the ten 100-lb lead weights, channel numbers for floor-level accelerometers of interest, and placement of the 5 subfloor foam blocks. (Not to scale.)

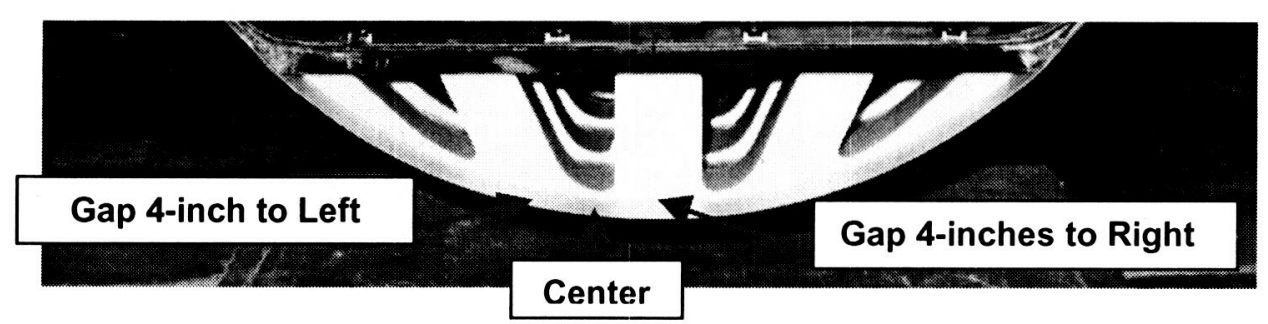

Figure 3. Locations of center, 4 inches left of center, and 4 inches right of center.

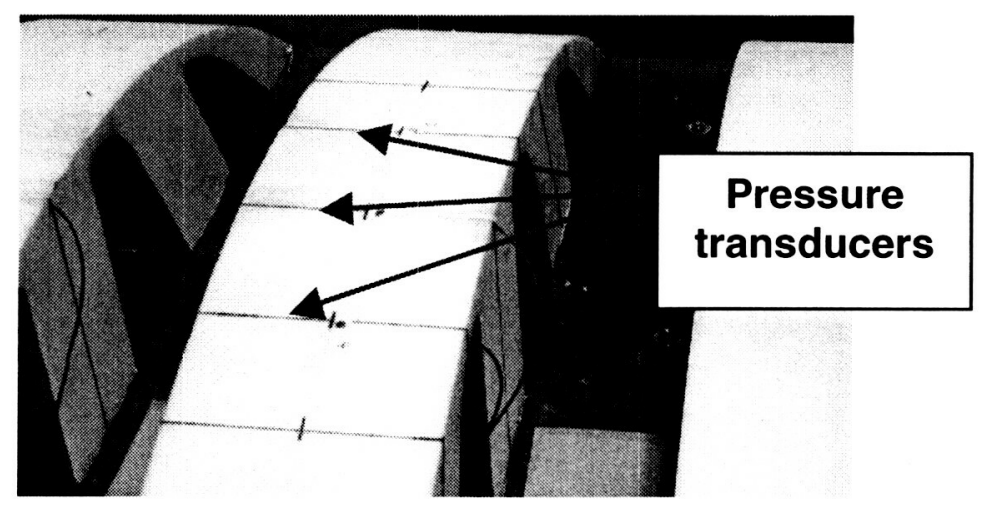

Figure 4. Close-up picture of center-block pressure gage installation. 


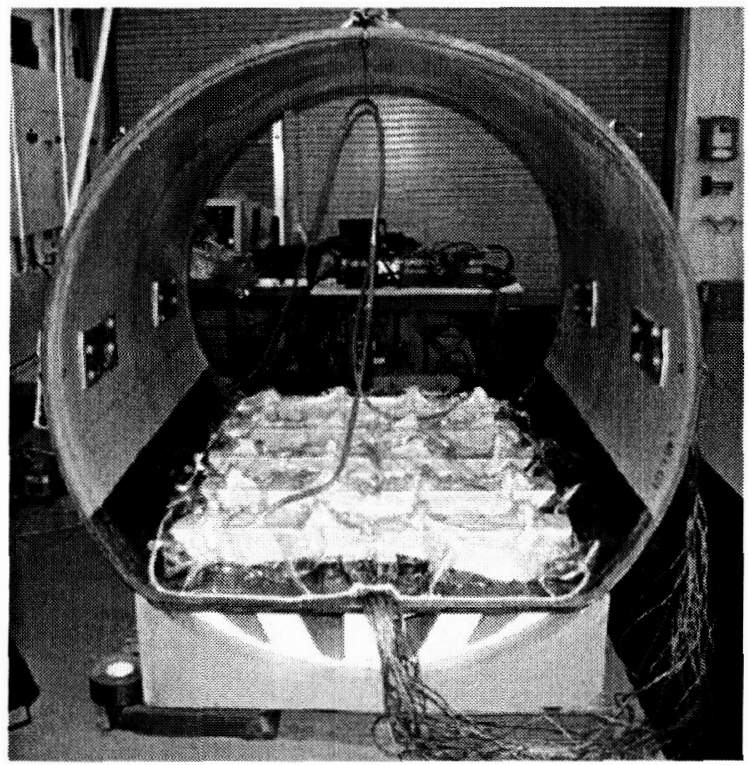

Figure 5. Vacuum bag enclosing lead blocks, transducers, and cables.

\section{Summary of Test Results}

\section{Structural Damage}

A video frame of the fuselage section impacting the water is shown in Figure 6. Following the test, the fuselage section was retrieved from the pool and the section was inspected for damage. Post-test examination of the subfloor region indicated considerable damage to the outer skin. The center "finger" of all five foam blocks showed no sign of crushing, and there was also very little debonding of the face sheets from the foam. A post-test view of the bottom of the section taken from the front is shown in Figure 7, in which the five Rohacell foam blocks can be distinguished. Note that the right side suffered more damage than the left. It is not known whether all of the damage occurred during the initial impact of the section into water. Some damage could have occurred on the secondary impact when the section hit the bottom of the pool. The unsupported areas of the outer skin between the Rohacell blocks showed the most damage. No damage was observed to the floor and upper fuselage cabin region.

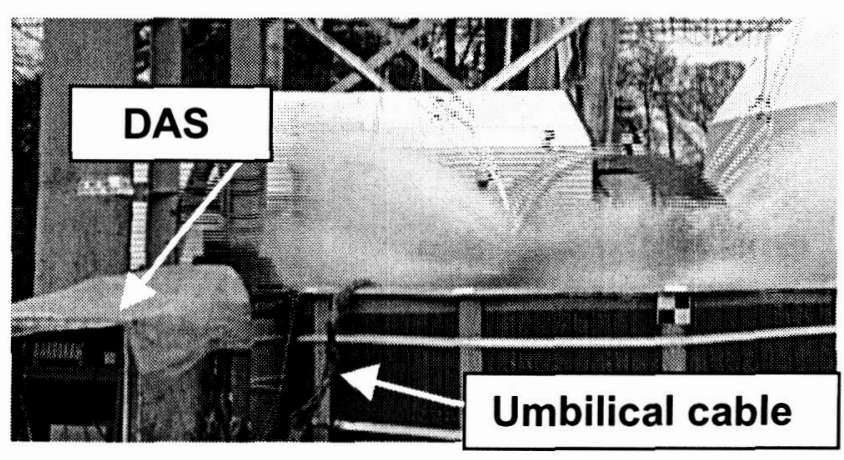

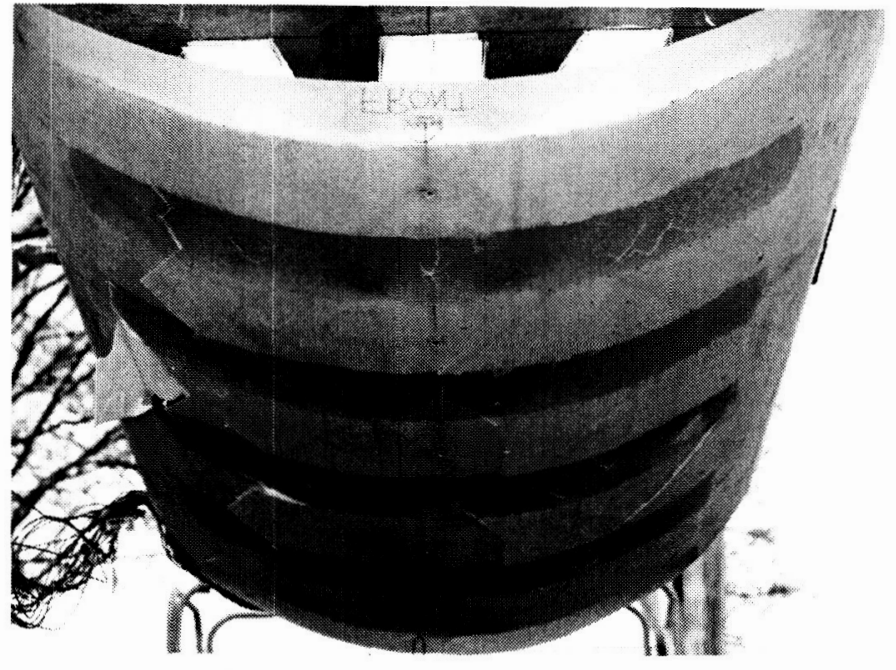

Figure 7. Bottom view of the fuselage section post-test showing damage.

A photograph of the subfloor from the bottom is shown in Figure 8 . The thin outer portions of the foam blocks were loaded in a radial direction by the water impact. Since these outer fingers are weak in this direction, the foam cracked and the face sheets debonded due to bending, as is observed in Figure 8 .

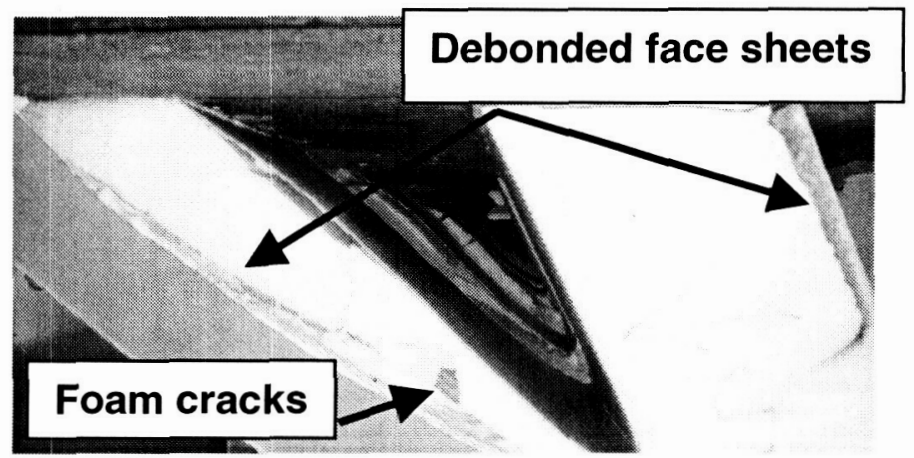

Figure 8. Post-test rear view of subfloor showing foam cracks and face sheet debonding.

\section{Pressure time histories}

The pressure transducers were the first instruments to detect the fuselage impact with the water. The section was pitched slightly front-end down at water contact, and the resulting time difference between the peak front-end pressure transducer on block 1 and the rear transducer on block 5 is approximately 3 milliseconds, as shown in Figure 9. By filtering the data with an SAE Channel Filter Class (CFC) 180 low-pass digital filter [14], the short-duration 90 - 100 psi initial peak dynamic pressure, seen in the unfiltered data, is reduced to approximately 45 - 50 psi. These high pressures are only maintained for 2-4 milliseconds before dropping to approximately $20 \mathrm{psi}$. After 0.01 seconds, the pressure reaches an approximate steady-state value around 5-10 psi.

Figure 6. Photograph of the splash at impact. 


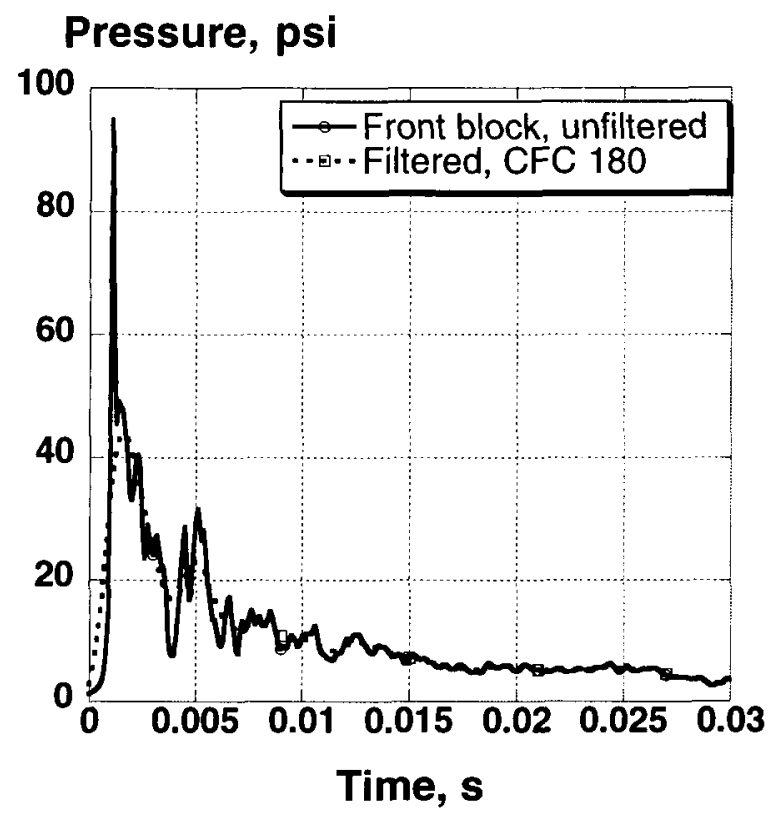

(a) Front pressure transducer.

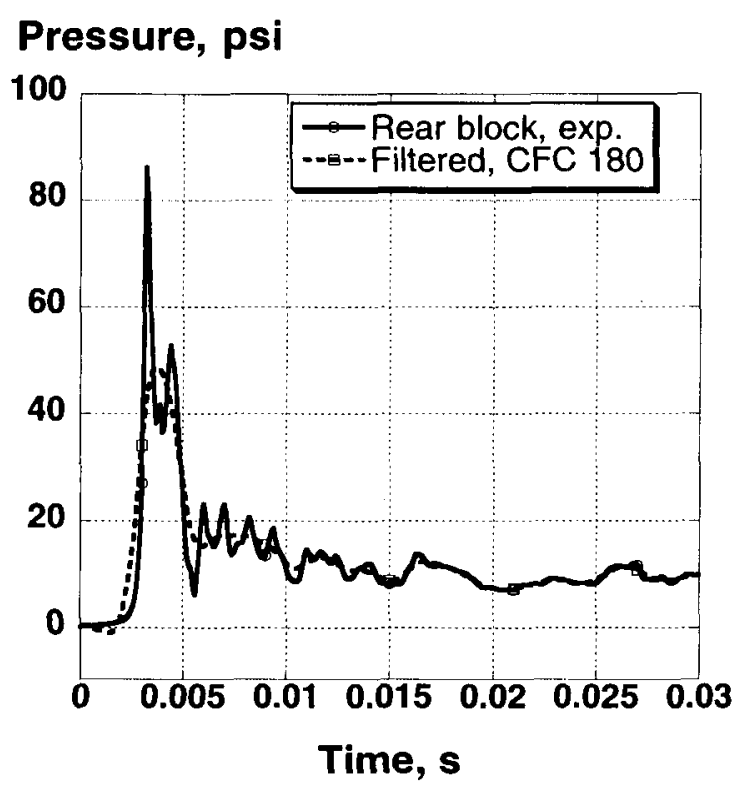

(b) Rear pressure transducer.

Figure 9. Pressure data recorded from the front and rear pressure transducers.

\section{Floor-level acceleration time histories}

Acceleration time histories are presented for the front and rear inboard and outboard locations on the 100-lb lead weights, whose locations are shown in Figure 2. Unfortunately, channel 1 was inoperative. Consequently, data from an accelerometer attached directly to the seat rail (channel 2) is presented instead. Another reason for using channel 2 is to illustrate the high amplitude, high frequency data from an accelerometer attached directly to the seat rail. Due to observed left-right symmetry, the acceleration responses from the left side will be emphasized in the paper.

Since filtering typically distorts the experimental data to some degree, plots of the raw and filtered floor-level acceleration responses are shown in Figure 10 for channels 2, 10, 9, and 18, corresponding to the inboard and outboard positions on the left side of the fuselage floor at the front and rear, respectively. The floor-level acceleration data were filtered with an SAE CFC 60 low-pass filter [14]. Unlike the three other accelerometers, the accelerometer denoted channel 2 was not attached to a lead block, but was instead mounted to the seat rail on the floor. In Figure 10 (a), the raw and filtered data for channel 2 is shown to the same scale as for the other plots to enable a good view of the filtered response. However, the same plot is reprinted in Figure 11 showing the maximum amplitude of the raw data. Obviously, the unfiltered acceleration data at location 2 contains high-frequency vibrations of much higher magnitude than the data obtained from accelerometers located on the lead masses, as seen by comparing the responses shown in Figures 10 and 11.

By viewing the plots in Figure 10, it is apparent that the onset rate of the filtered acceleration is less than the onset rate for the raw data. Note that all of the acceleration traces in Figure 10 start from $-1 \mathrm{~g}$ since the section was in free-fall before impact. The data in Figure 10 also illustrate the differences in the overall shape and magnitude of the acceleration responses as a function of channel location. The outboard acceleration pulses are of slightly higher magnitude than the inboard responses, and the pulse has a half sine wave shape with a single peak. The inboard acceleration pulses have two peaks with the first of higher magnitude than the second. In general, the inboard and outboard acceleration responses do not vary greatly as a function of longitudinal position, e.g., the front and rear inboard acceleration responses shown in Figures 10 (b) and (d) are similar.

In Figure 12 (a), a comparison of the pressure at the bottom of the first (front) foam block with the floor-level vertical acceleration from channel 33 , the right front outboard location, shows that the peak pressure occurs much earlier than the peak acceleration of the lead block. The acceleration from channel 33 is integrated to obtain the fuselage section velocity time history, shown in 12 (b). This plot shows that the section comes to rest after 0.5 seconds. The initial acceleration pulse from 0 to 0.05 seconds only removes about half of the initial impact velocity. 


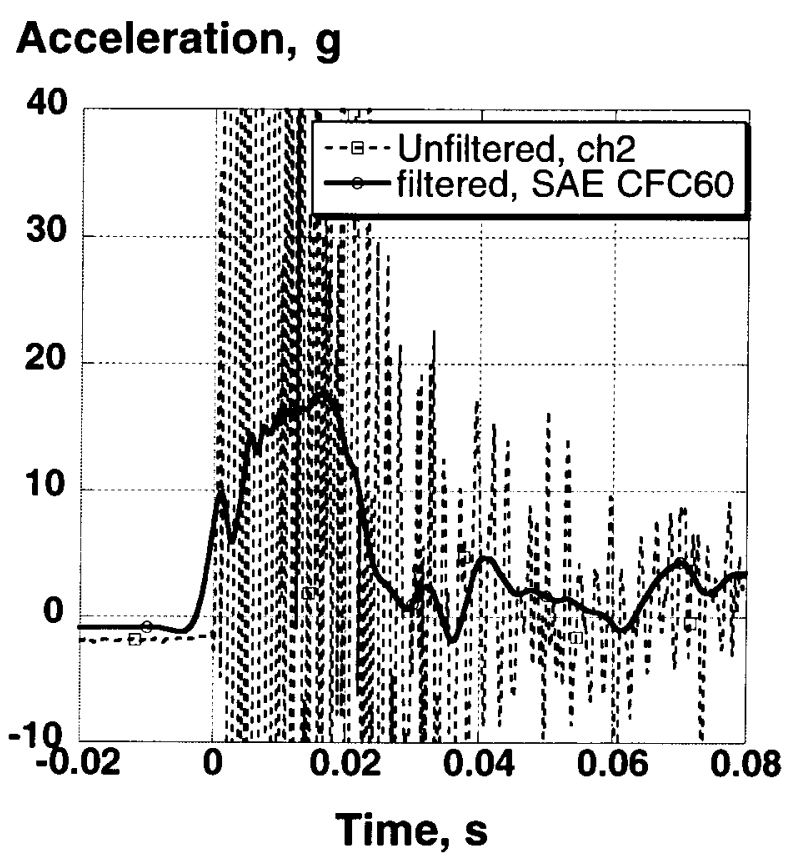

(a) Left front outboard, channel 2

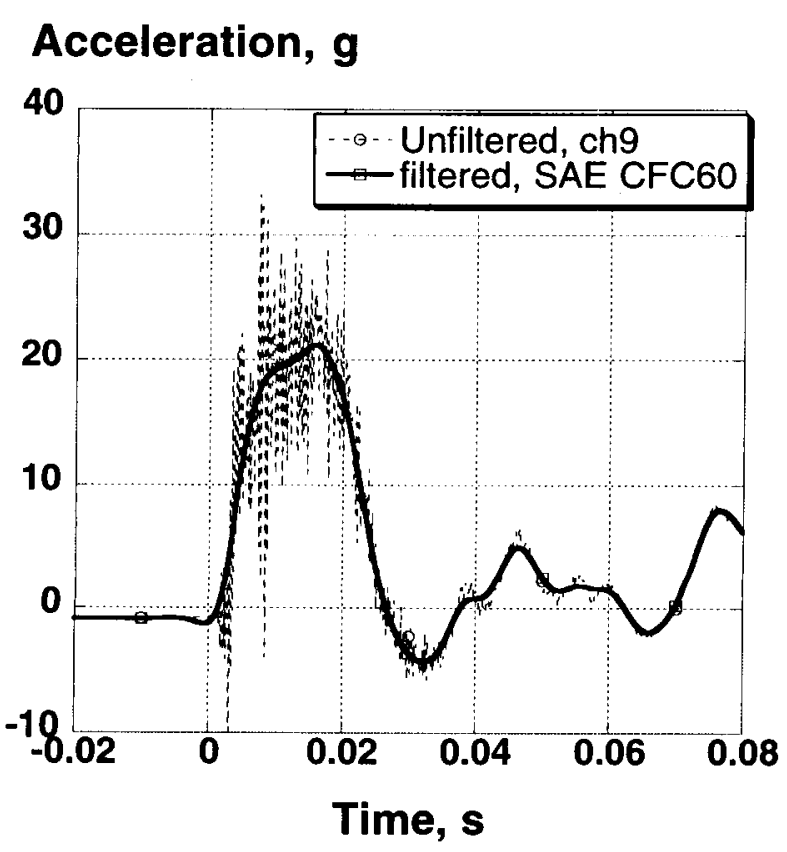

(c) Left rear outboard, channel 9.

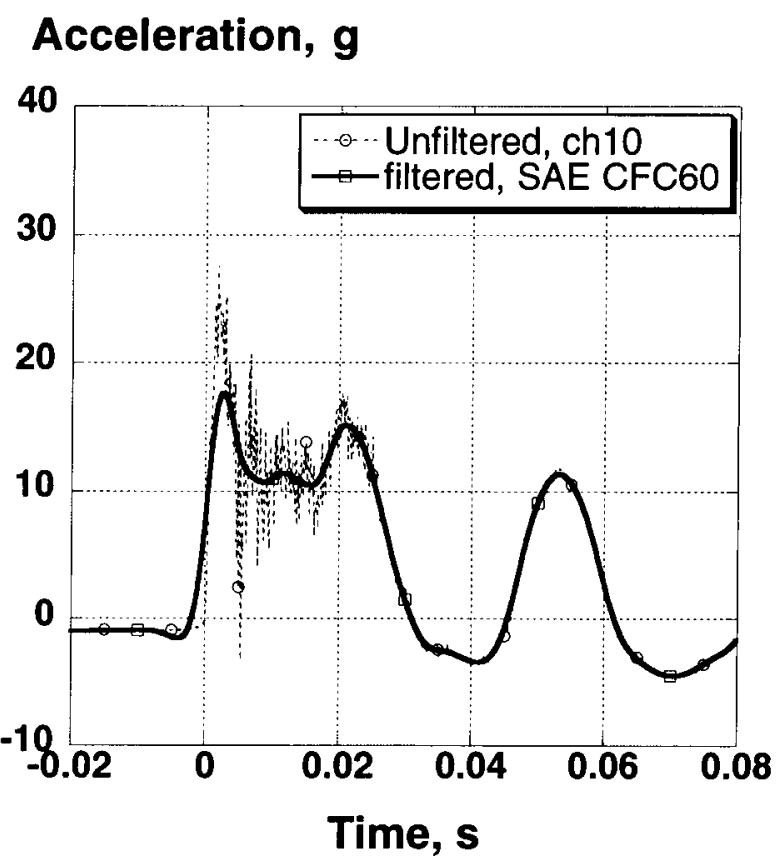

(b) Left front inboard, channel 10.

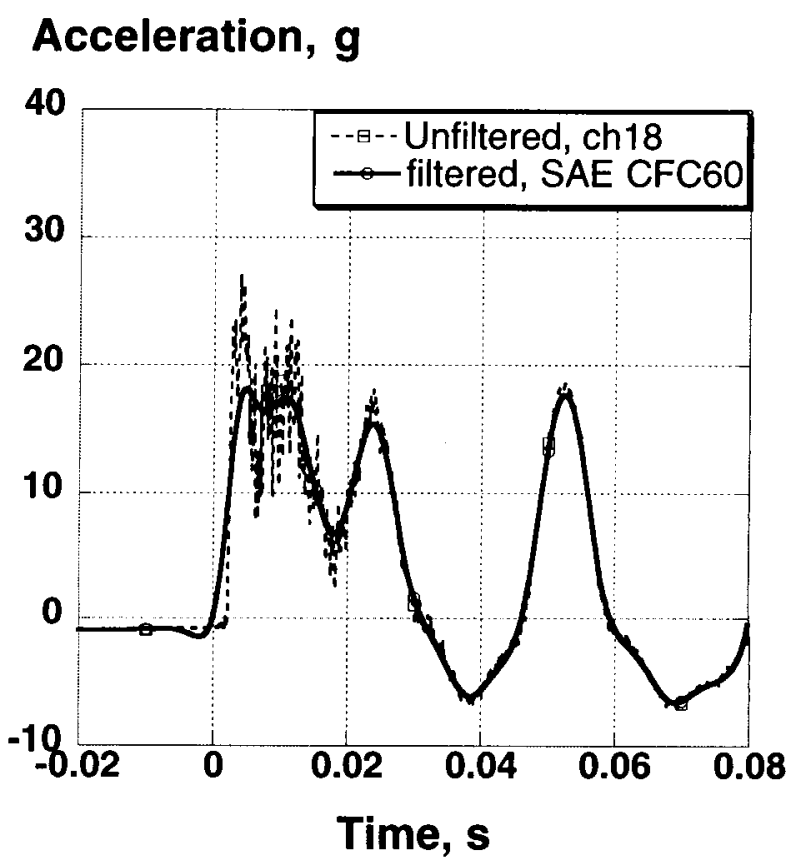

(d) Left rear inboard, channel 18

Figure 10. Comparison of unfiltered and filtered inboard and outboard accelerations for front and rear locations. Due to left and right symmetry, only locations on the left side are shown. 


\section{Acceleration, $\mathbf{g}$}

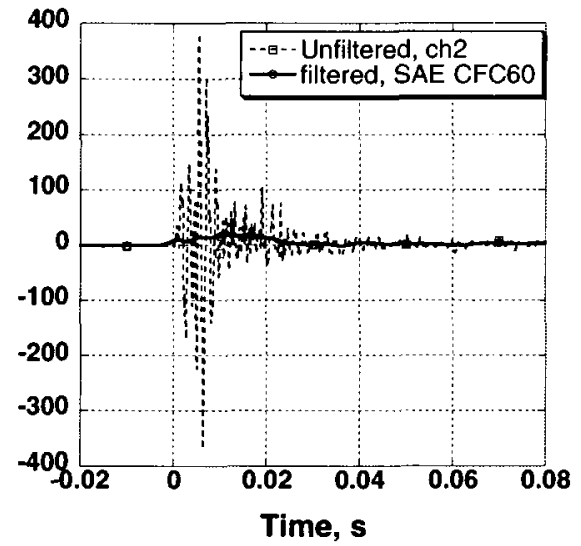

Figure 11. Raw and filtered acceleration traces for seatrail mounted accelerometer, channel 2 .

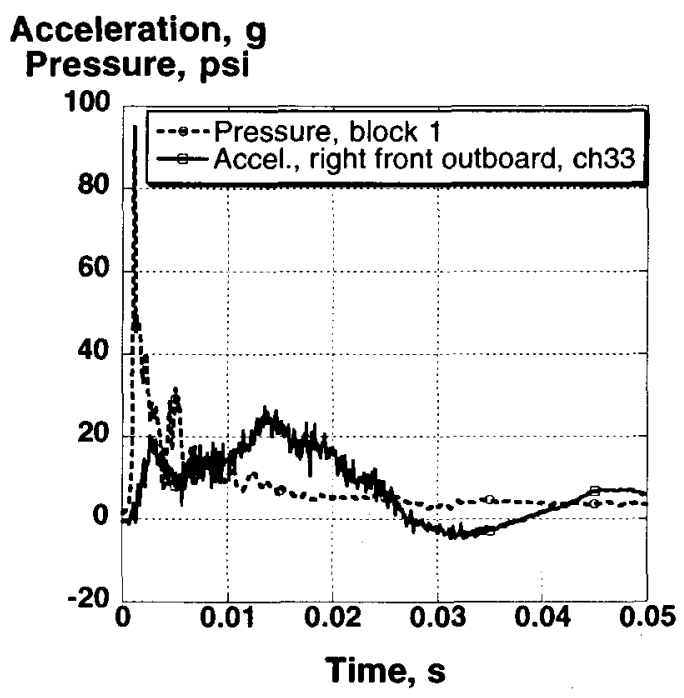

(a) Pressure and acceleration near front of section.

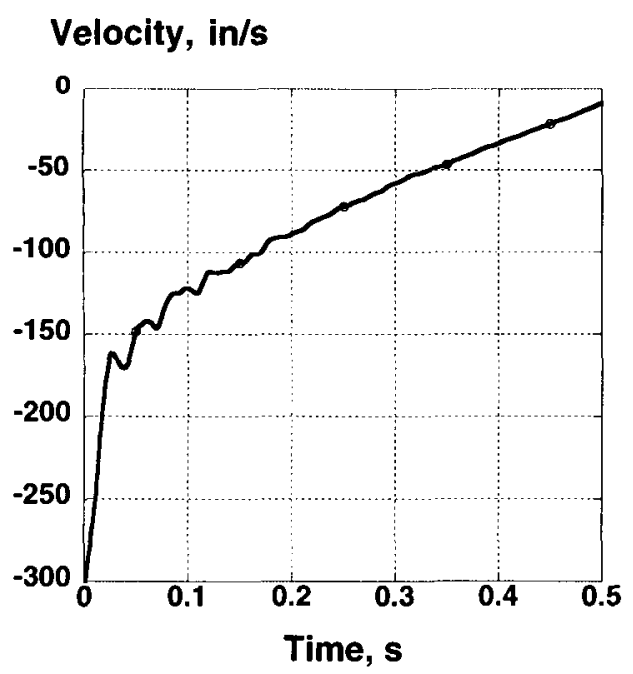

(b) Velocity time-history front of section.

Figure 12. Pressure on foam block 1; and acceleration and velocity time-histories for channel 33 at the right front outboard location.

\section{Analysis of Water Impact}

von Karman Closed-form Solution

In an early National Advisory Committee for Aeronautics (NACA) paper published in 1929 [15], von Karman derived a formula for the maximum pressure on a wedge impacting water. The formula was derived to help designers of sea planes and sea plane floats to calculate the stresses during landings. The formula for maximum pressure on a cylindrical wedge with an angle alpha between the water and one plane of the wedge is given by

$$
P_{\max }=\rho v_{0}^{2} \pi \cot \alpha
$$

where $\rho$ is the density of water, $v_{0}$ is the sink speed of the aircraft, and $\alpha$ is the angle between the water and the lower surface of the wedge. Using the impact velocity of approximately $25 \mathrm{ft} / \mathrm{s}$, equation (1) was used to develop the curve shown in Figure 13 for the maximum pressure as a function of the wedge-angle, alpha. The bottom of the 5-ft. cylindrical fuselage, if approximated by a wedge shape, would be expected to have a shallow angle with the water of between 5 and 15 degrees, corresponding to pressures of between 50 and $150 \mathrm{psi}$. The peak values measured by the pressure gages fall within this range (see Figure 9).

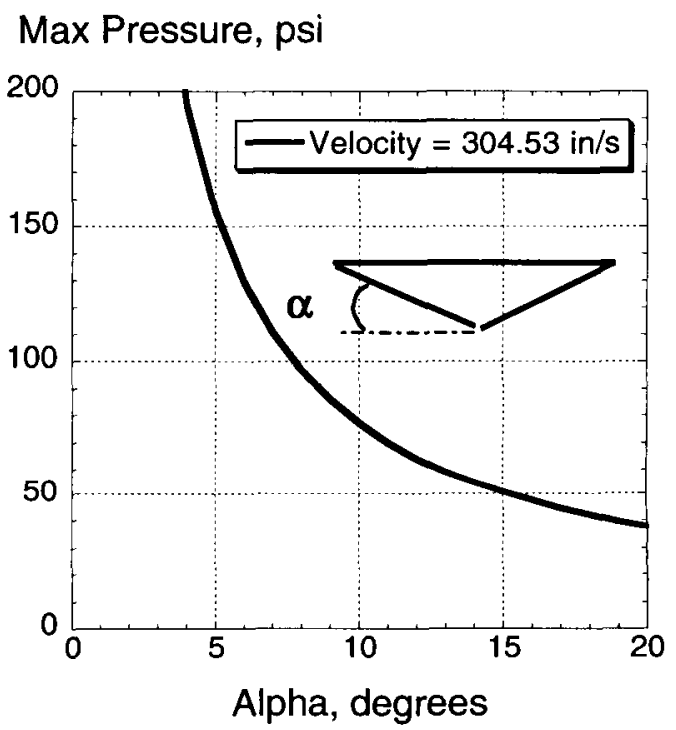

Figure 13. Maximum pressure versus the angle, $\alpha$, for a wedge impacting water with a velocity of $304.5 \mathrm{in} / \mathrm{s}$.

Modeling of Water Impact with Nonlinear Dynamic Finite Element Codes

In the design phase, numerical modeling is the most logical and least expensive approach to study the effects of impact on a structure. With the advent of fast inexpensive workstation computers, numerical modeling of the impact of an aircraft structure into a fluid media such as water can now be modeled with explicit nonlinear dynamic codes. In this investigation, a variety of techniques, including pure Lagrangian and coupled 
Largrangian-Euler methods, were investigated to model water impacts in two codes frequently used in crash analysis, LS-DYNA [16] and MSC.Dytran [17]. In addition, the size, shape, and discretization of the water and air (void) volume was varied to determine if these parameters would affect the test-analysis correlation. The density of the water and equation of state were also varied to study the sensitivity to these parameters.

Typically, the aircraft structure is modeled with a Lagrangian mesh composed of deformable elements with associated nodes that move with the element. The fluid is typically modeled using a stationary Eulerian mesh in which the fluid material flows, while conserving mass, momentum, and energy. When using an Eulerian fluid solver, a portion of the air volume above the water must also be modeled with an Eulerian mesh to allow the wave to form.

In the past, the modeling of materials such as fluids and gases that have little or no shear strength using a Lagrangian approach was only practical for a very short time before the mesh degenerated into small or negative volumes. Even with remeshing, the lack of shear strength led to instabilities. However, with the advent of smooth particle hydrodynamic ( $\mathrm{SPH}$ ) techniques, both the structure and fluid can be modeled using a Lagrangian approach. The SPH technique is a meshless Lagrangian method to model fluids. In the SPH method, material is treated as particles that have their masses smoothed in space. SPH computes strain rate and stress divergence based on the nearest neighbors of a particle. The SPH method has been implemented in LS-DYNA, and it was used in this study.

Various schemes are used to model the fluid meshes and their interaction with the structural mesh. In MSC.Dytran, the meshes can interact by either general coupling or by the Arbitrary Lagrangian-Eulerian (ALE) coupling. In general coupling, a coupling surface, which must form a closed volume, is created on the Lagrangian mesh. The coupling surface calculates the action-reaction forces and then applies them to the material in the Lagrangian structure and to the Eulerian fluid to provide the fluid-structure interaction. MSC.Dytran models using general coupling run from 50 to 90 percent faster using the "fast" coupling algorithm, which is a special case of general coupling. The fast coupling algorithm requires that the Euler mesh be orthogonal and perfectly aligned with the global coordinate system. In the ALE formulation, the Eulerian mesh and the Lagrangian mesh are coupled through an ALE interface surface. The Lagrangian and Eulerian grid points in the interface surface coincide in physical space but are separate in logical space. The ALE interface moves as the Lagrangian structure deforms. Thus, the Eulerian mesh boundary moves. The fluid material flows through the Eulerian mesh as in general coupling; however, the mesh can also be made to follow the structure by defining the
Eulerian grid points as ALE grid points. Typically, the ALE formulation is more computationally efficient than the general coupling method. However, the deformation of the structure at the ALE interface should be relatively smooth. Note, that smoothness does not require the deformation to be small in magnitude.

\section{MSC.Dytran Lagrange-Euler Model Development and Correlation Studies}

Since the MSC.Dytran general coupling fluid-structure interaction model requires a closed coupling surface around the Langrangian structure, dummy triangular shell elements were added to the section model to close off both ends. The closed coupling surface was defined as the outside of the cylinder plus the two ends as shown in Figure 14. Excluding the dummy triangular shell elements, the fuselage model had 32,404 elements consisting of shell, solid, and beam elements. The original fuselage model was created in MSC.Dytran. Additional details about the fuselage model can be found in References 6 - 8 .

The first water impact model used 6-inch cubic Euler elements to fill a water region $12 \mathrm{ft}$. $\times 12 \mathrm{ft}$. $\times 3.5 \mathrm{ft}$. deep. The Euler "air" volume was 24 cubes wide, 24 cubes long, and two elements deep with a total of 1,152 elements; while the water volume was 24 cubes wide, 24 cubes long, and 7 elements deep with 4,032 elements. The air was actually modeled as empty volume or void. This Euler void region is needed so that a wave can be generated above the water surface level. The second model filled the same Euler void and water volumes with 3-inch solid elements. The two models are shown in Figure 14. Both MSC.Dytran models were run using the "fast" general coupling algorithm. Since the fast coupling algorithm requires that the Euler mesh be orthogonal and perfectly aligned with the global coordinate system, the cylindrical geometric shape of the physical pool could not be used. However, the exact geometry is not likely required as long as there is a sufficiently large Euler region as compared with the test specimen (see next section for more discussion). The models were run with MSC.Dytran 2002 Intel Linux Release (July 31,2001 ) on a Linux workstation using one Intel Xeon processor with a clock speed of $2.0 \mathrm{GHz}$. The model with the 6-in. Euler mesh required approximately 6.5 CPU hours to run 0.07 seconds real time. The initial time step of 1.99 microseconds was controlled by a small quad element in a fillet area where the floor meets the side of the fuselage. The final time step was controlled by the subfloor foam and was reduced to under a microsecond. The refined model with the 3 -inch Euler mesh required 12.2 CPU hours to run 0.07 seconds. The second simulation was more stable as the time step at the end of the run was 1.56 microseconds. 


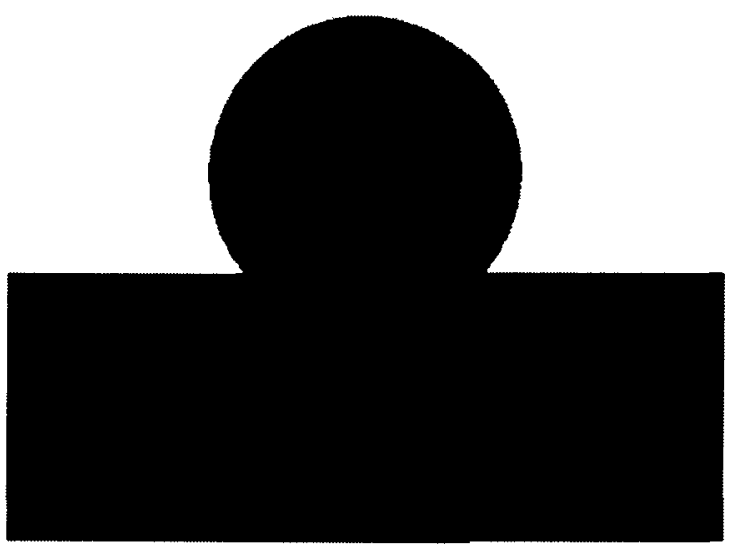

(a) 6-in. Euler mesh.

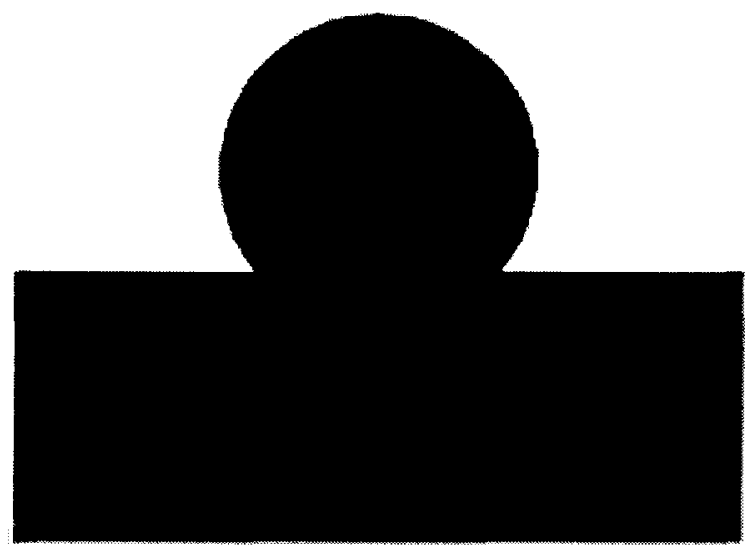

(b) 3-in. Euler mesh.

Figure 14. The MSC.Dytran models having two different Euler air and water meshes.

A comparison of the MSC.Dytran predictions for the two symmetrically located left inboard accelerometers on the front and rear 100-lb. lead blocks that were mounted on the fuselage floor are shown in Figure 15. The experimental data and the analytical predictions were filtered with a digital filter based on the SAE CFC 60 specifications [14]. In general, the predicted accelerations match the experimental waveform, but exceed the experimental values. The MSC.Dytran simulation predictions for the two approximately symmetric outboard locations are shown in Figure 16. Recall that data from channel 1 was lost. Thus, data from channel 2, which was located on the floor near to channel 1, was shown instead. Consequently, the response is not entirely the same. The analytical predictions from the 3-in. Euler model were closer in magnitude and provided a better match to the overall shape of the experimental acceleration responses than did the results from the 6-in. Euler model. In general, the models predicted the differences in shape between the inboard and outboard accelerations well. Since the current version of MSC.Dytran does not allow for failure of the coupling surface due to the closed surface general coupling requirement, neither of the
MSC.Dytran models incorporated failure of the lower fuselage skin.

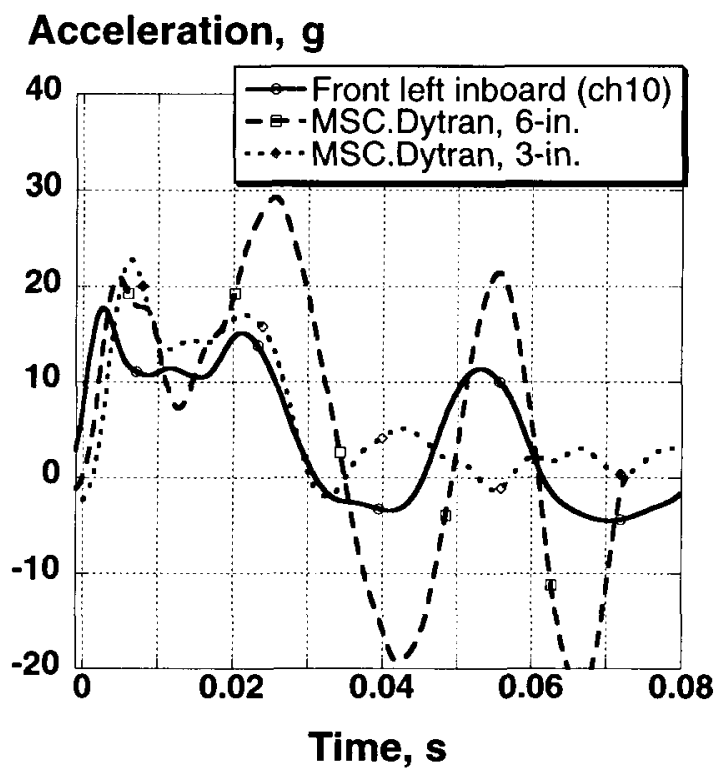

(a). Front left inboard, channel 10

\section{Acceleration, $\mathbf{g}$}

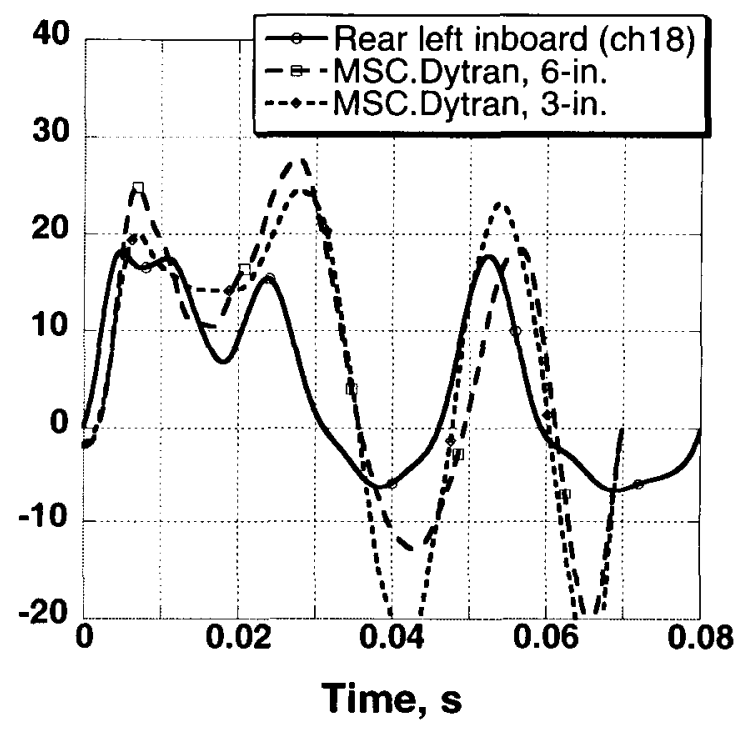

(b). Rear left inboard, channel 18.

Figure 15. Filtered experimental acceleration responses compared with MSC.Dytran predicted inboard accelerations for the 6-in. and 3-in. Euler meshes. 


\section{Acceleration, $\mathbf{g}$}

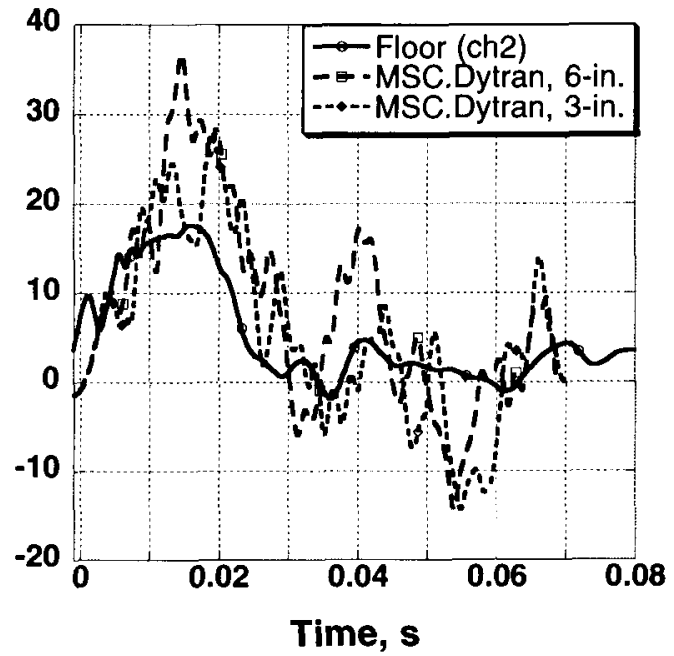

(a) Front left outboard floor-level, channel 2.

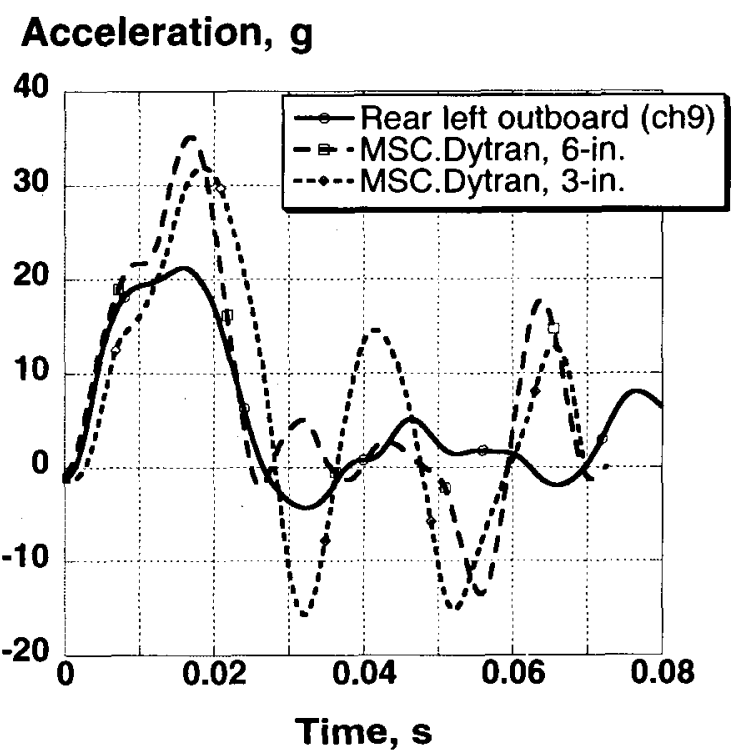

(b) Rear left outboard, channel 9.

Figure 16. Comparisons of MSC.Dytran predicted outboard accelerations with experiment for the 6 -in. and 3-in. Euler mesh.

Other authors have reported that beyond a critical depth, width, and length of the Euler water mesh, the initial acceleration pulse results do not vary significantly. A depth of approximately 3 - $\mathrm{ft}$. was reported as the "critical depth" for the drop of a helicopter section into water at around $25-36 \mathrm{ft} / \mathrm{s}$ [18]. For the present study, two water depths were modeled in MSC.Dytran. The shallow case was 5 cubes deep or 2.5 feet, while the larger depth was 7 cubes deep or 3.5 feet, the actual depth of the pool. No significant difference in the acceleration pulse for the first 0.03 seconds could be detected, as shown in Figure 17.
Acceleration, $\mathbf{g}$

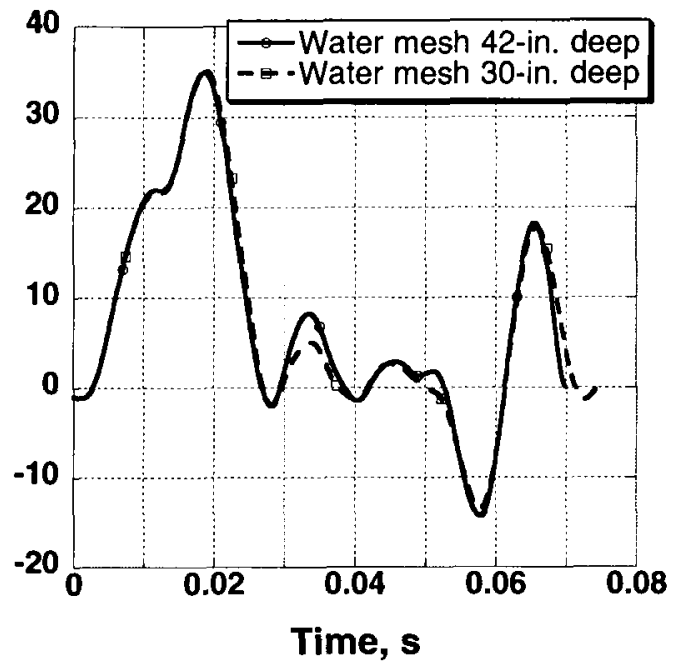

Figure 17. Effect of model water depth on predicted acceleration at the rear left outboard location.

\section{LS-DYNA Model Development and Correlation Studies}

A series of LS-DYNA models were constructed to study the effects of the water and air characterization. The LS-DYNA fuselage model was converted from the original MSC.Dytran model and was as identical to the MSC.Dytran model as possible. The same fuselage model was used for each case study, except for the final run during which the lower fuselage skin was allowed to fail. Since failure is catastrophic and may have a major effect on the simulation, no failure strains were assigned to the elements forming the lower skin for the early simulations. The parameters that were varied to evaluate the test-analysis correlation were the shape of the water and air region and the discretization and the equation of state of the water, including viscosity effects.

The first model of the water (and air layer above the water) used a relatively coarse uniform 6-inch rectangular Euler mesh. Coupon data for an angle-ply layup similar to the outer skin at the bottom of the section indicated that failure would likely occur for a plastic strain of from 2 to 3 percent. When a failure strain of 2 percent was applied to the lower skin for the model with the 6-in. Euler mesh, no flow of water occurred since the Euler mesh was too large compared with the size of the elements of the skin bottom. Consequently, the Euler mesh was rediscretized to contain uniform 3 -inch cubic solid elements. To assess the effect of shape, cylindrical volumes representative of the pool geometry were constructed. The final Euler mesh used a gradient with the smallest 1-inch elements located in close proximity to the bottom of the fuselage section. When failure strains were applied to this model, water did flow through the areas formed by the deleted failed elements. In the final simulation, the water was modeled using the Lagrangian SPH method. The initial models were run using LS-DYNA version 960. Some of the later models were run with the beta version 970 , which allows pressures to be extracted across the Euler interface more efficiently. Since LS-DYNA allows for viscosity, runs were made with a 
viscosity coefficient of $\mu=2.57 \mathrm{e}-07$ psi-second in the water equation-of-state [19]. However, the effect of viscosity was barely detectable in the filtered acceleration responses. Consequently, all results shown are without viscosity. In addition, models with small density variations for water (fresh to salt water) were constructed and run. The effect of small variations in density was also found to be negligible. Post processing including filtering was accomplished using the software package LS-POST.

\section{LS-DYNA models with rectangular 6-inch and 3-inch Euler meshes}

All LS-DYNA fuselage models consisted of 32,404 elements. In contrast to the MSC.Dytran water volume, which was $12 \mathrm{ft}$. $12 \mathrm{ft}$. $\times 3.5 \mathrm{ft}$, the water volume in LSDYNA model was $15 \mathrm{ft}$. $\times 15 \mathrm{ft}$. $3.5 \mathrm{ft}$ deep. The first LS-DYNA model, which used 6-inch cubic elements to represent the water and void, consisted of a total of 37,833 nodes and 41,404 elements. The water mesh was 7 elements deep and $30 \times 30$ elements in width and length for a total of 6,300 elements. The Euler void mesh was 3 elements deep with a total of 2,700 elements. To study convergence, a more refined Euler mesh was next constructed with 3-inch cubic elements. Since each dimension was halved, there were 8 times as many Euler elements in the refined mesh for a total of 72,000 elements. The total number of elements (including the section) was 108,004. The ALE coupling method was used for all the LS-DYNA models that had an Euler fluid representation. Also, unlike MSC.Dytran, LS-DYNA does not require a closed coupling surface between the Euler and Lagrangian elements, so no dummy shell elements were required to close the open ends of the section. Front views of the models having the 6- and 3-in. Euler meshes are shown in Figure 18.

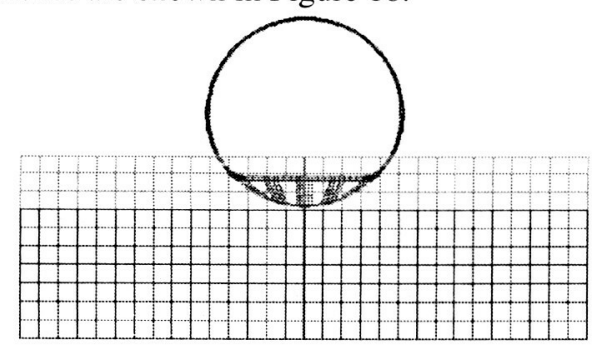

(a). Front view of the 6-in. Euler mesh

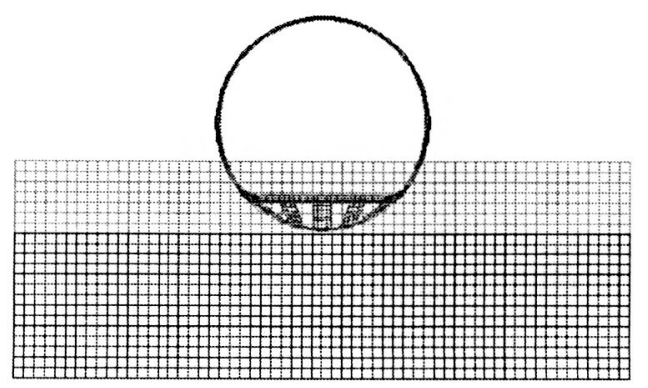

(b). Front view of the 3-in. Euler mesh.

Figure 18. Views of the LS-DYNA model with the 6- and 3-inch uniform Euler mesh.

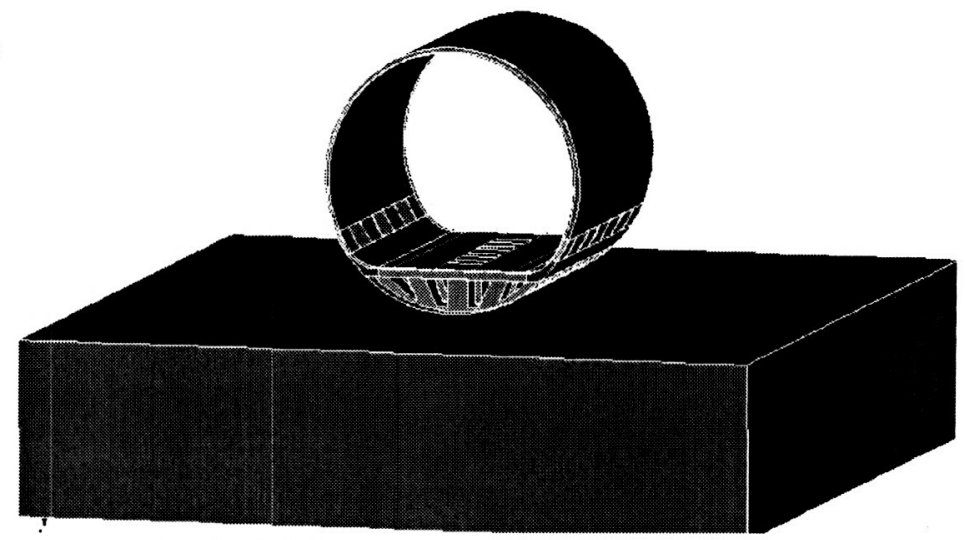

(a) Shaded three-quarter view of the model.

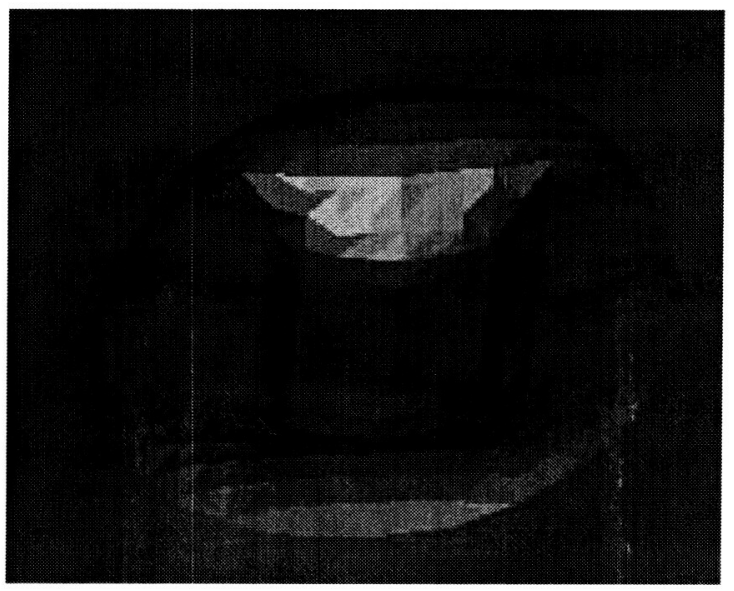

(b) Developing wave at time 0.080 seconds.

Figure 19. Three-quarter view of model and development of "splash" at time 0.080 seconds for the 6-inch uniform Euler mesh.

A shaded three-quarter view of the model is illustrated in Figure 19 (a). The displacement of the water into the void for the 6-in. Euler model at the end of the simulation is shown in Figure 19(b). The front and rear left inboard and outboard acceleration traces were selected for comparison of the model predictions with the experimental data, as shown in Figures 20 and 21. As with the MSC.Dytran results, the accelerations were overpredicted. However, the shapes of the acceleration responses and the pulse durations were well tracked. Comparisons of the LS-DYNA predicted accelerations for the same inboard and outboard locations indicate that the magnitudes of the acceleration responses are lower for the 3-in. Euler mesh and are closer to the experimental results. For an equivalent model, the time-step calculated by LS-DYNA was about one-tenth the size of the time step calculated by MSC.Dytran. Consequently, an LS-DYNA model required about $4-5$ times the CPU time on the same Linux workstation. 


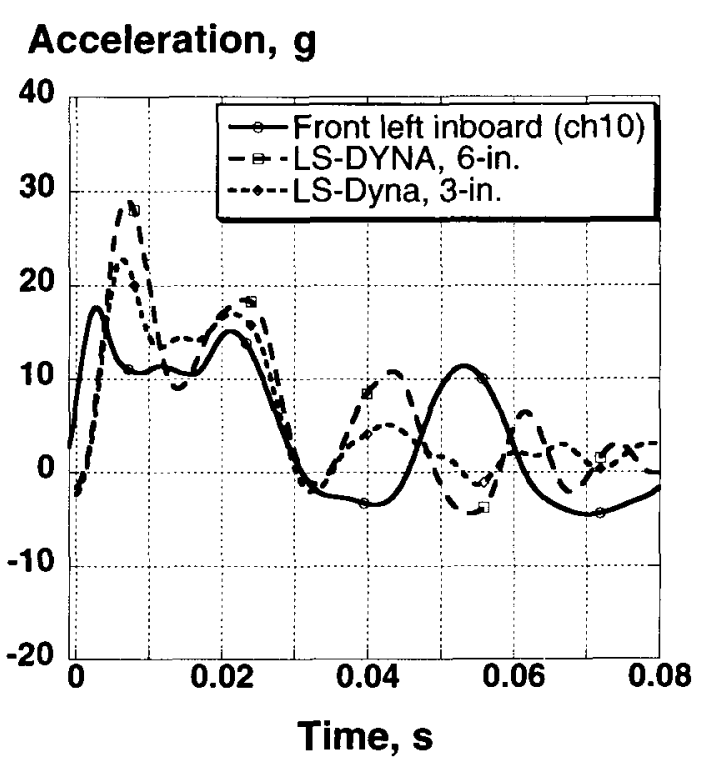

(a). Front left inboard, channel 10.

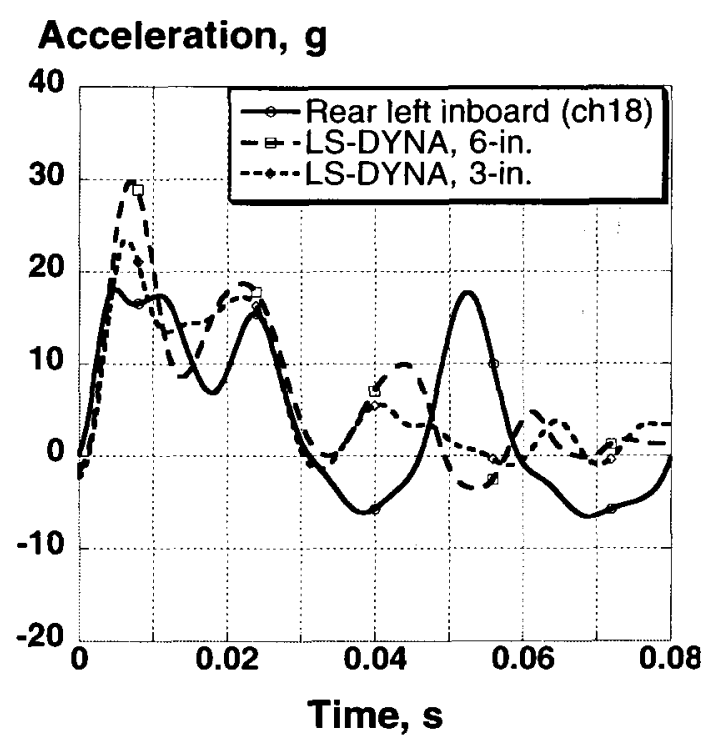

(b). Rear left inboard, channel 18.

Figure 20. Filtered experimental acceleration responses compared with LS-DYNA predicted inboard accelerations for the 6- and 3-in. Euler meshes.

\section{LS-DYNA model with 15-ft. diameter cylindrical Euler} region

In order to study the effect of the geometric shape of the water, a model was constructed that matched the geometry of the $15-\mathrm{ft}$. diameter pool. Each Euler element was two inches deep, but with dimensions approximately 3 -inch $\times 3$-inch in plane varying to fit the circular geometry. This model consisted of 105,304 elements of which 62,900 were Euler elements. A front and side view of the discretization of the water pool plus air is shown in Figure 22. The total number of elements in the Euler region is just slightly less than the total in the model with the rectangular 3-inch mesh, shown in Figure 18. However, the mesh is more refined in the vertical direction. The comparison of experimental and predicted acceleration responses for the left inboard and outboard locations are shown in Figures 23 and 24 , respectively. The predicted results for the cylindrical Euler model correlate better with the experiment than those from the uniform 3-in. Euler model, shown in Figures 20 and 21. However, the improved correlation may be due to the slightly smaller Euler elements beneath the fuselage and the 2-in. depth of each element, and not the actual cylindrical shape of the Euler region.

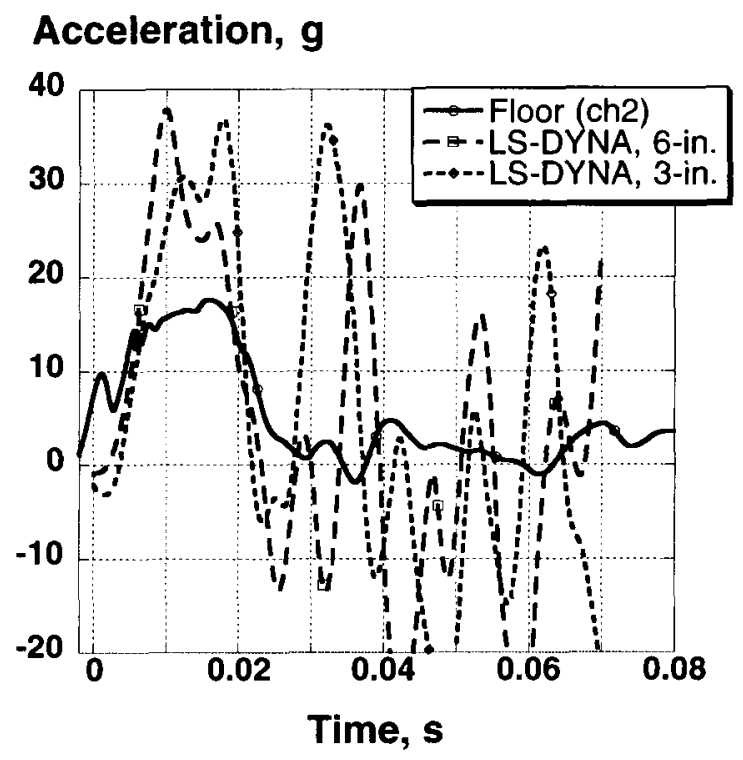

(a) Front left outboard floor-level, channel 2.

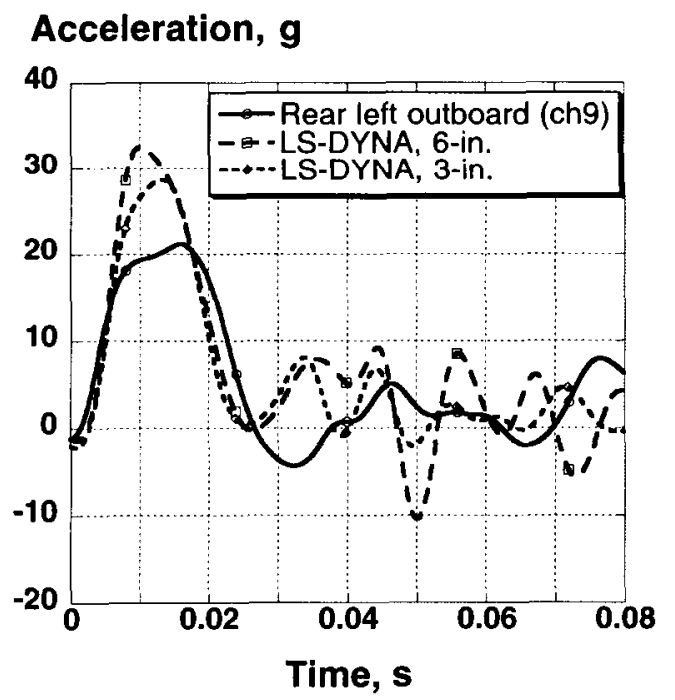

(b) Rear left outboard, channel 9.

Figure 21. Filtered experimental acceleration responses compared with LS-DYNA predicted outboard accelerations for the 6- and 3-in. Euler meshes. 


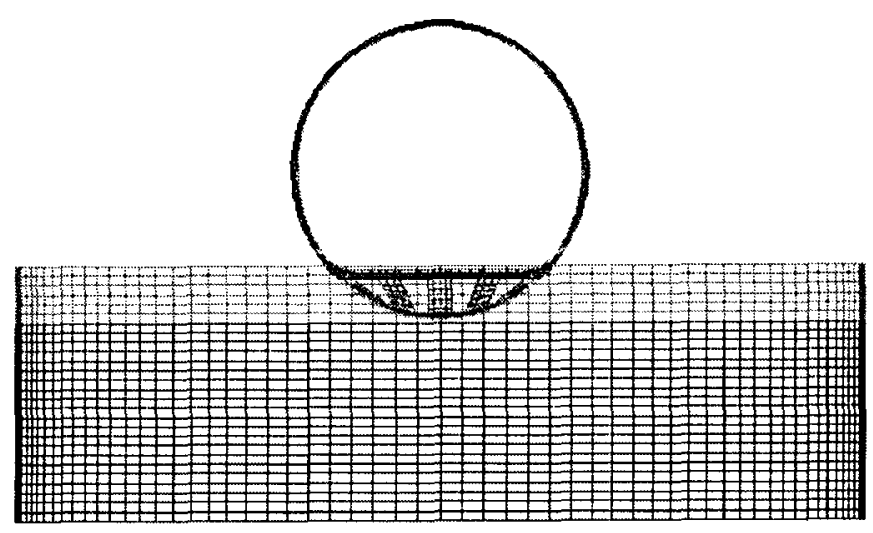

(a) Side view.

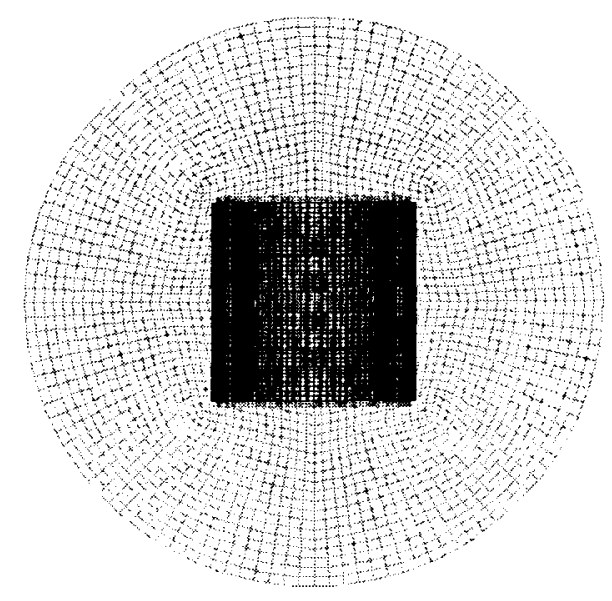

(b) Top view.

Figure 22. Side and top view of the cylindrical Euler air (light) and water (dark) region.

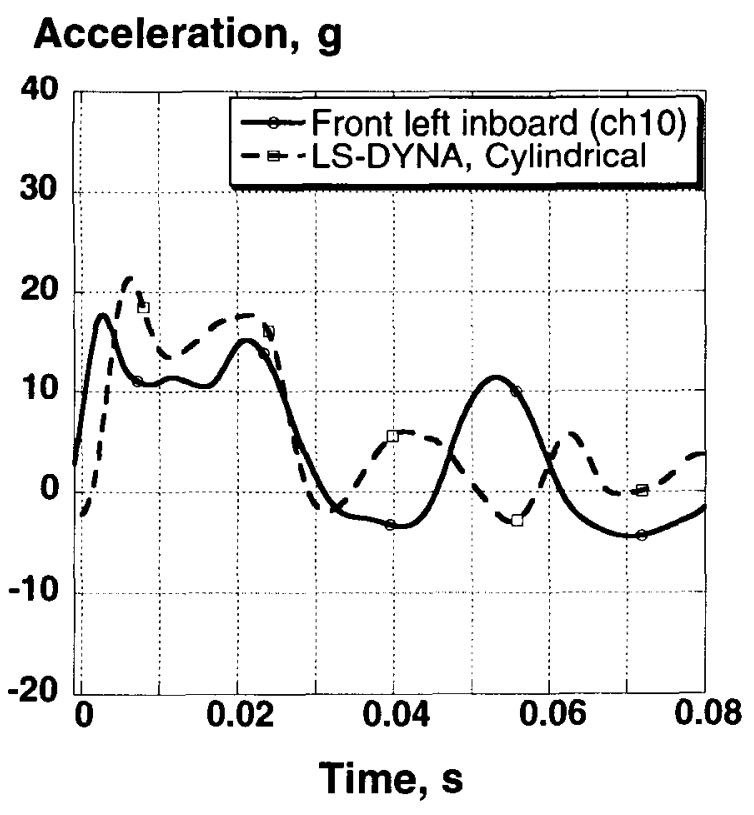

(a) Front left inboard, channel 10.

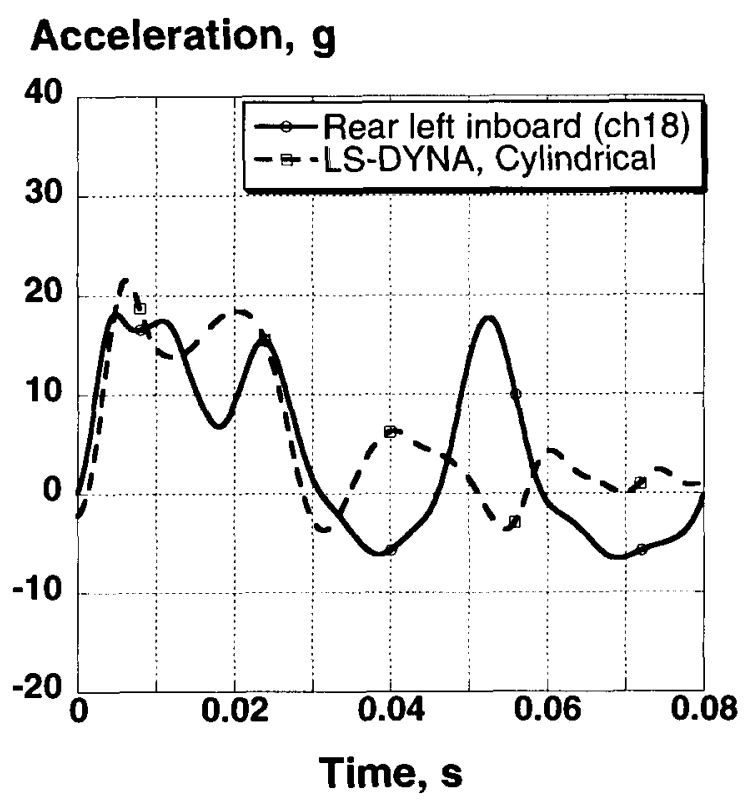

(b) Rear left inboard, channel 18.

Figure 23. Filtered experimental acceleration responses compared with LS-DYNA predicted inboard accelerations for the cylindrical Euler mesh.

\section{LS-DYNA 1-inch gradient mesh}

The final Euler model was executed with a refined gradient mesh in which the region beneath the fuselage section contained 1-in. cubic solid elements. A "slice" of the model is shown in Figure 25. The total number of elements in the model is 122,404 and the number of solid elements in the Euler mesh is 90,000. Comparisons of experimental and predicted acceleration responses for the same four channels representing the left inboard and outboard positions on the front and rear lead blocks are shown in Figures 26 and 27, respectively. The first peak acceleration of $27 \mathrm{~g}$ 's for the front inboard locations exceeds the measured value of 18 . However the second peak is very close to the measured values of approximately $18 \mathrm{~g}$ 's.

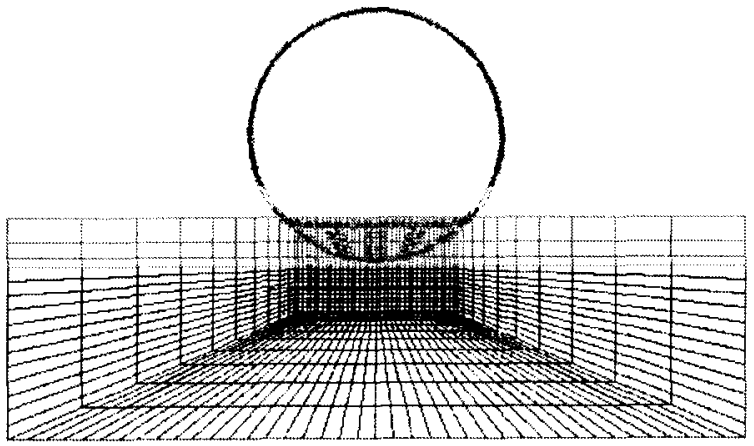

Figure 25. Front view of a slice of the gradient-mesh that becomes a refined 1-inch mesh beneath the fuselage section.

The initial peak acceleration measured for the rear inboard location is approximately $18 \mathrm{~g}$ 's as compared with the 
predicted value of $19 \mathrm{~g}$ 's. The rear outboard position prediction of $23 \mathrm{~g}$ 's compares quite favorably with the

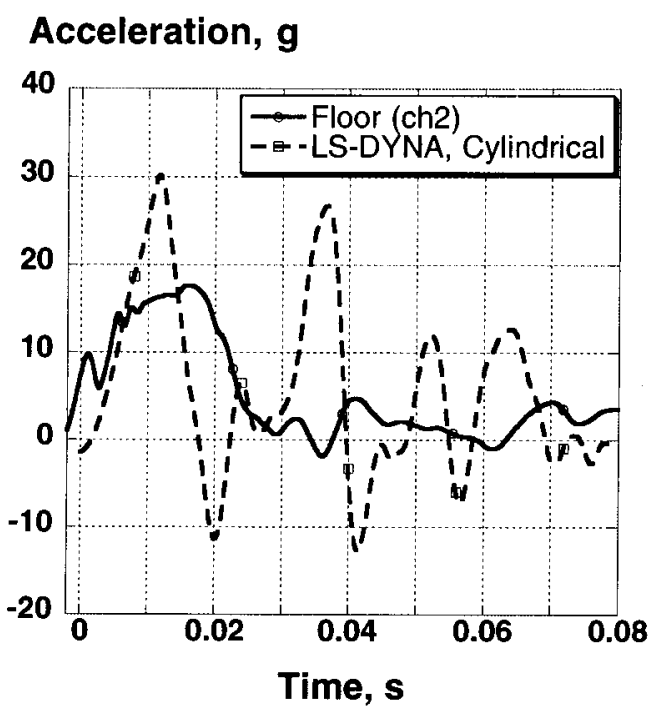

(a) Front left outboard floor-level, channel 2

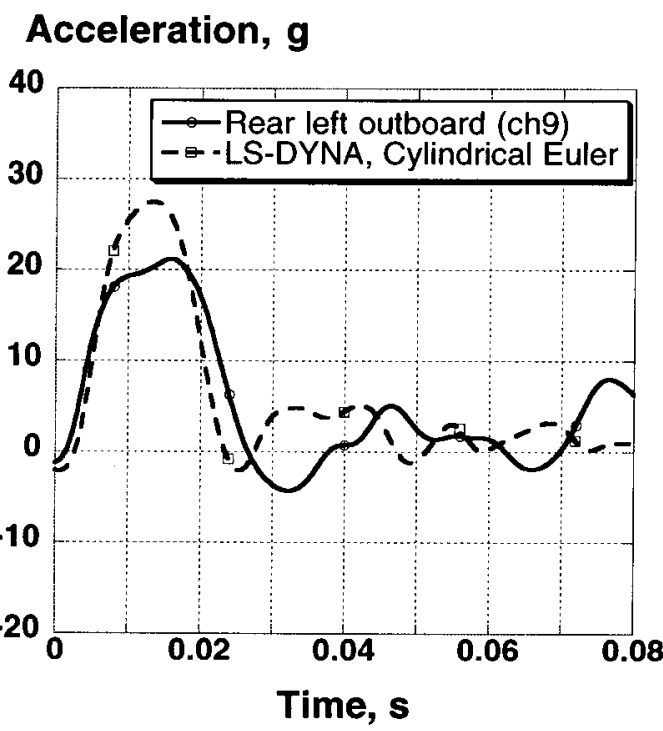

(b) Rear left outboard, channel 9.

Figure 24. Filtered experimental acceleration responses compared with LS-DYNA predicted outboard accelerations for the cylindrical Euler mesh.

measured value of $21 \mathrm{~g}$ 's. The only position that does not show good agreement is for the floor position (channel 2). The acceleration response for channel 2 was again highly oscillatory. Note that damping was not used in the fuselage model.

Pressure data was output from the LS-DYNA model with the 1-in. gradient Euler mesh for comparison with the experimental data, as shown in Figure 28. The predicted pressure for the front, bottom pressure transducer was calculated using the average of the applied pressure from the Euler mesh to the four shell elements of the outer skin connected to the node nearest to the location of the pressure transducer. The experimental pressure was filtered with an SAE CFC180 digital filter [14]. Note, the averaging process tends to effectively filter the predicted information. The predicted pressure response follows the experimental data very closely as shown in Figure 28. In order to obtain this information, the betaVersion 970 of LS-DYNA was required.

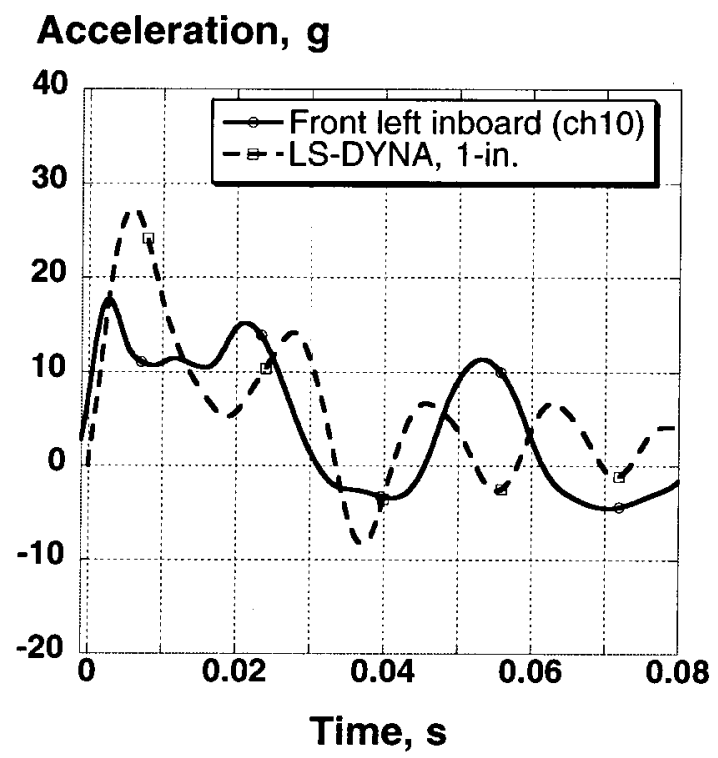

(a). Front left inboard, channel 10.

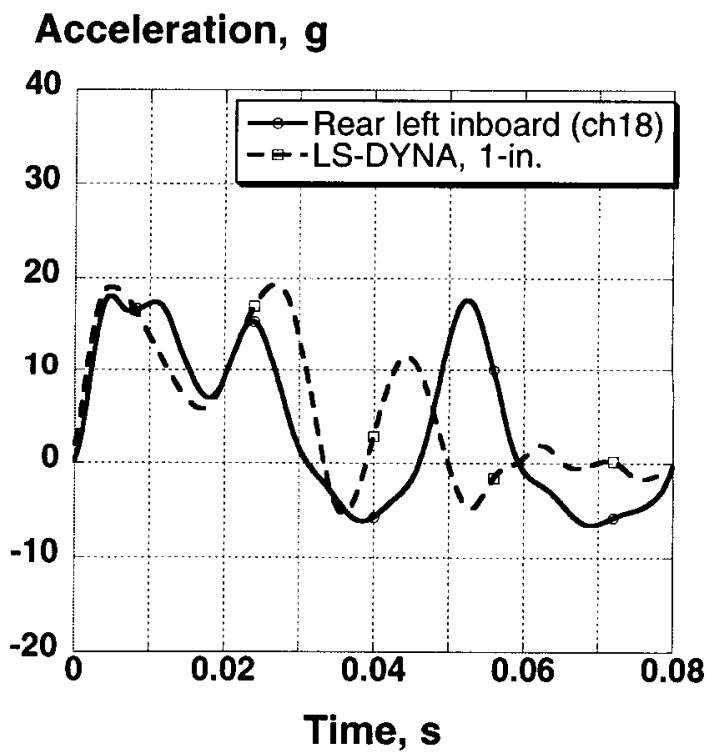

(b). Rear left inboard, channel 18.

Figure 26. Filtered experimental acceleration responses compared with LS-DYNA predicted inboard accelerations for the 1-in. gradient Euler mesh. 


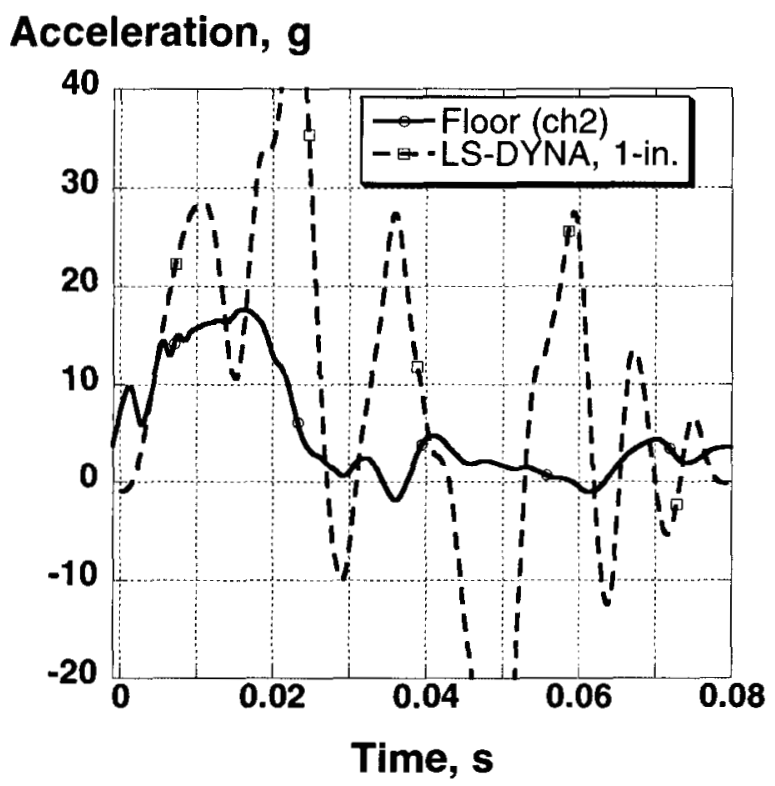

(a) Front left outboard floor-level, channel 2.

\section{Acceleration, $\mathbf{g}$}

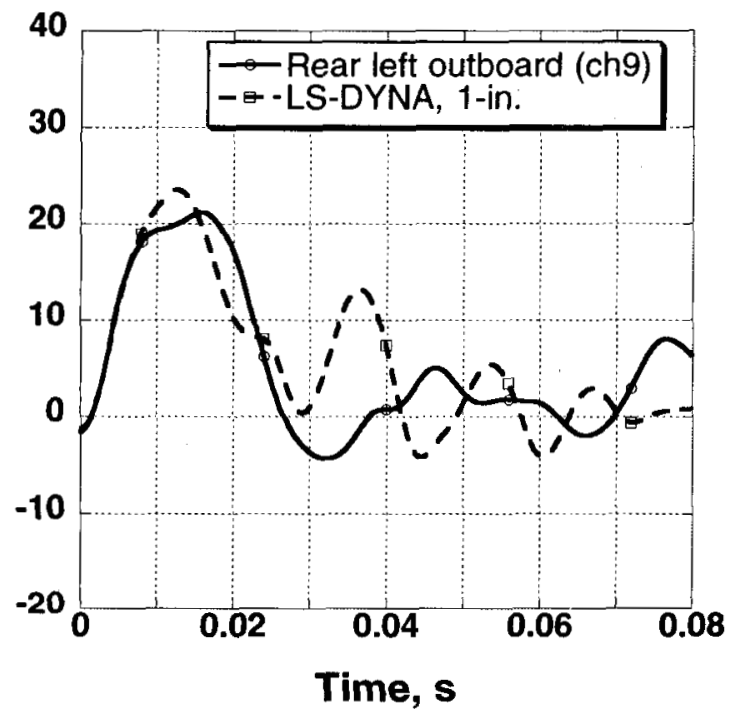

(b) Rear left outboard, channel 9.

Figure 27. Filtered experimental acceleration responses compared with LS-DYNA predicted outboard accelerations for the 1-in. gradient Euler mesh.

\section{Pressure, psi}

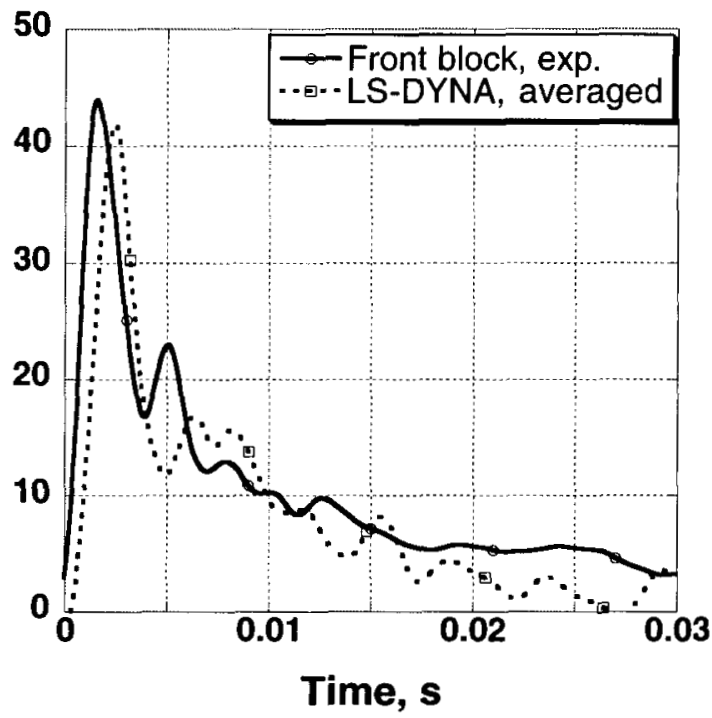

Figure 28. Comparison of the measured pressure on the first block with LS-DYNA averaged pressure.

LS-DYNA 1-in. gradient mesh with failure of fuselage subfloor $\underline{\text { skin }}$

The failure strain can be set on the material card for the bottom fiberglass skin to allow the elements to fail after a given strain is reached. When elements fail, they are deleted forming holes in the bottom surface that allow the water to flow through. The failure of the bottom skin is shown in Figure 29 for 0.01 seconds after impact. The left half of the figure shows the botiom skin of the fuselage viewed from an angle from above. In this case, the failure strain was set to 2 percent, which is a practical value for an angle-ply fiberglass laminate. The results show that the outer skin between the foam blocks fails catastrophically allowing the water to flow through as shown in the right side of the figure. Although the failure is dramatic, the initial peak accelerations were only reduced by a small amount from the original model without failure as shown in Figure 30. Since the run times for these models are long, the model with failure was only run long enough to capture the fundamental pulse; i.e., 0.04 seconds. The amount of damage predicted by this simulation was more severe than observed. Thus the simulated acceleration pulses with failure, shown in Figures 30 and 31, drop off too quickly after the initial peak due to the excessive failure. Since the actual strain-to-failure data for the angle-ply laminate was not available, the objective of specifying a failure strain was to determine the effect of failure on the simulation. The actual stress-strain curve to failure of the bottom skin laminate would be needed for a more accurate simulation. Also, note that when the failure strain criterion was met, the elements were deleted. Other failure options available in LS-LYNA such as "constrained tied nodes failure" were not run, but may reduce the severity of the damage. 


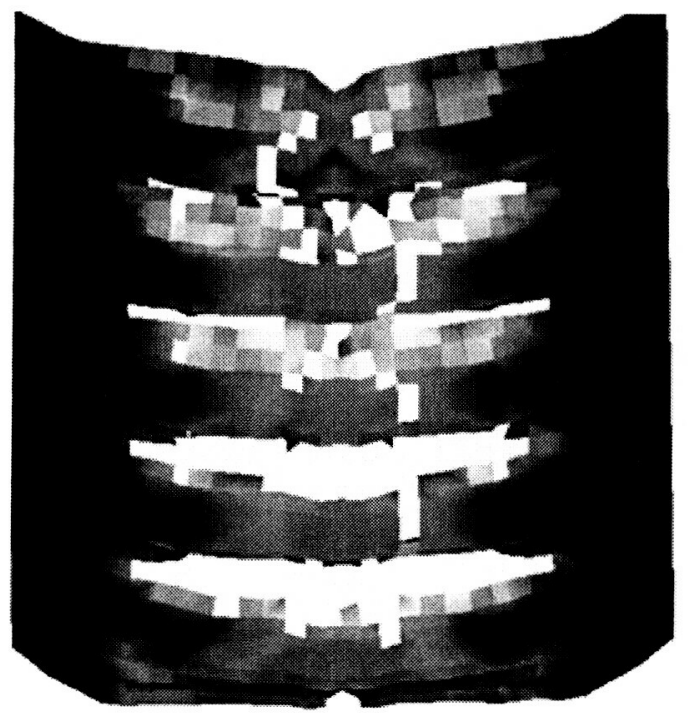

(a) Failures of the lower skin at time 0.01 seconds

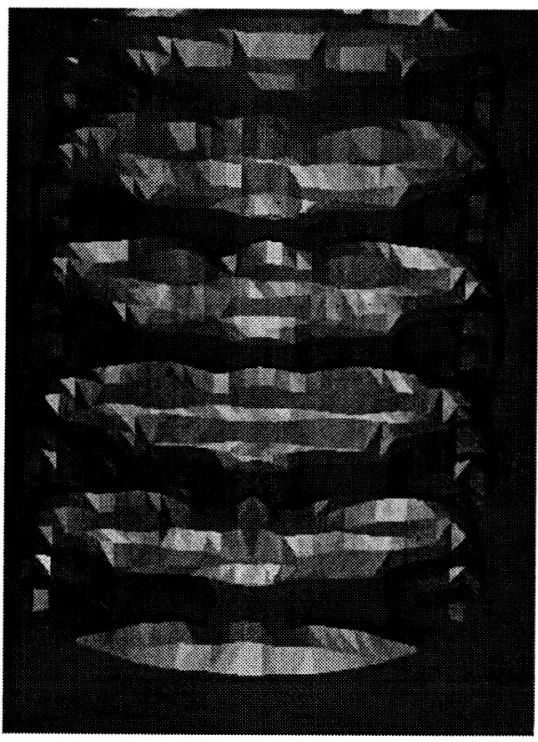

(b) Water flowing through the ruptured skin corresponding to 0.01 seconds

Figure 29. Computer graphics illustrating failures of the lower skin at time 0.01 second, which allow water to flow through the ruptured skin.

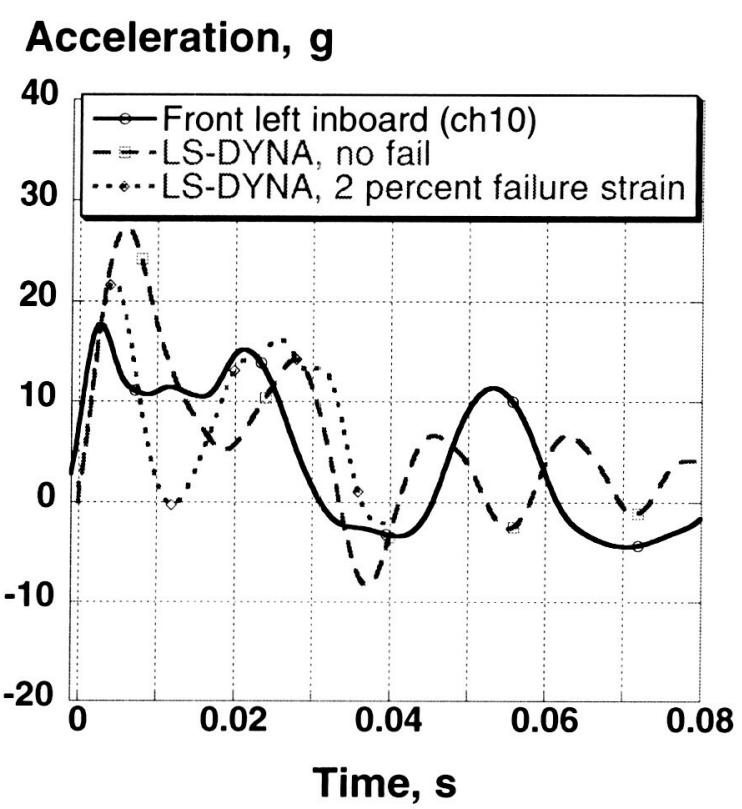

(a). Front left inboard, channel 10 .

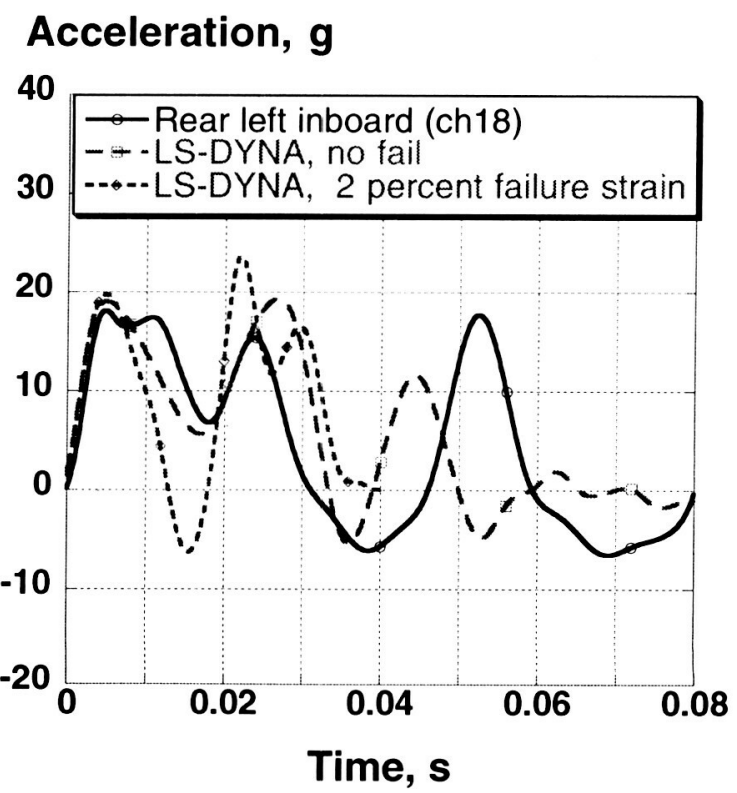

(b). Rear left inboard, channel 18.

Figure 30. Filtered experimental acceleration responses compared with LS-DYNA predicted inboard accelerations for the 1 -in. gradient Euler mesh, with and without failure. 


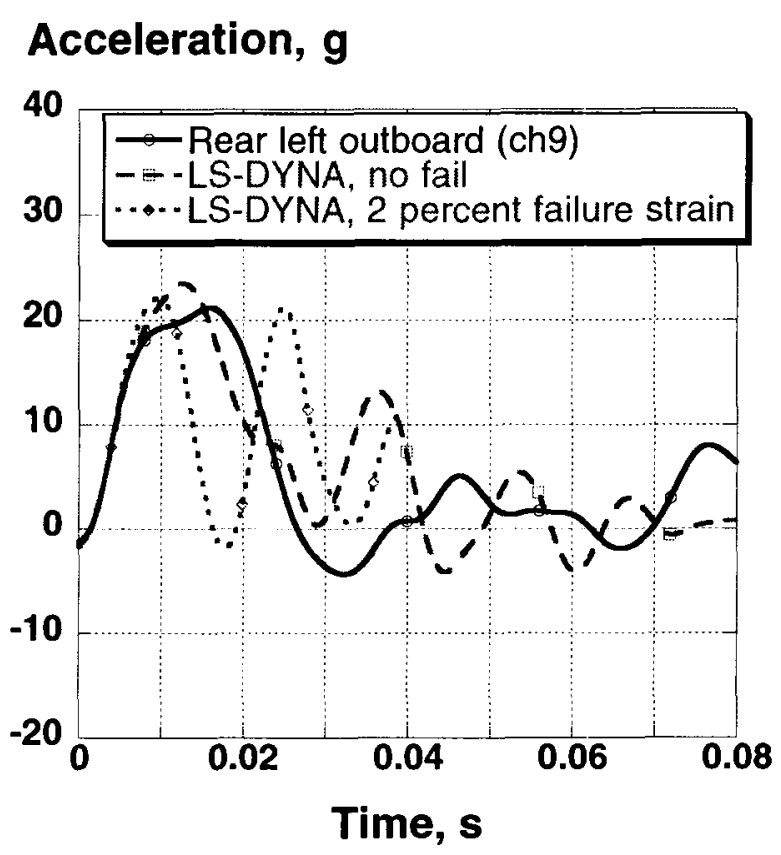

(a) Front left outboard floor-level, channel 2.

\section{Acceleration, $\mathbf{g}$}

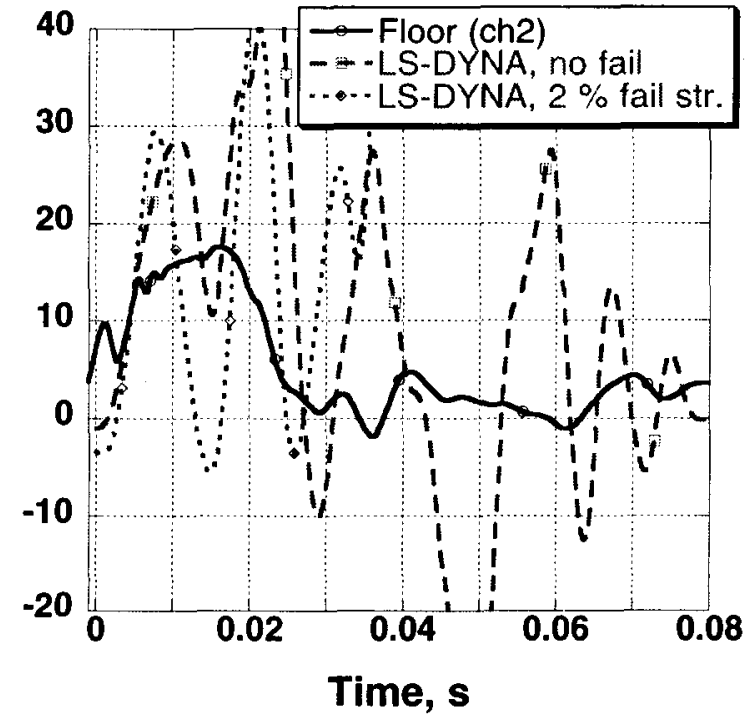

(b) Rear left outboard, channel 9.

Figure 31. Filtered experimental acceleration responses compared with LS-DYNA predicted outboard accelerations for the 1-in. gradient Euler mesh, with and without failure.

\section{LS-DYNA Smooth Particle Hydrodynamics (SPH)} Meshless Lagrangian Technique

The final LS-DYNA simulation was executed using the SPH meshless Langrangian technique. The model is depicted in Figure 32. Comparisons of experimental and predicted acceleration responses for the four channels representing the left inboard and outboard positions on the front and rear lead blocks are shown in Figures 33 and 34, respectively. As with the 1-in. gradient Euler model, the peak accelerations for the front and rear inboard locations are very close to the measured values of approximately $18 \mathrm{~g}$ 's. In addition, the peak acceleration measured for the rear outboard location compares well with the measured value of $22 \mathrm{~g}$ 's. The prediction for the floor accelerometer, channel 2, is shown in Figure 34 (a). Even when filtered, the predicted acceleration trace shows oscillations of higher frequency than measured experimentally. Other than this location, the SPH method was very successful in modeling the water impact. However, the SPH method required about $2-3$ times the CPU time as compared with the ALE approach.
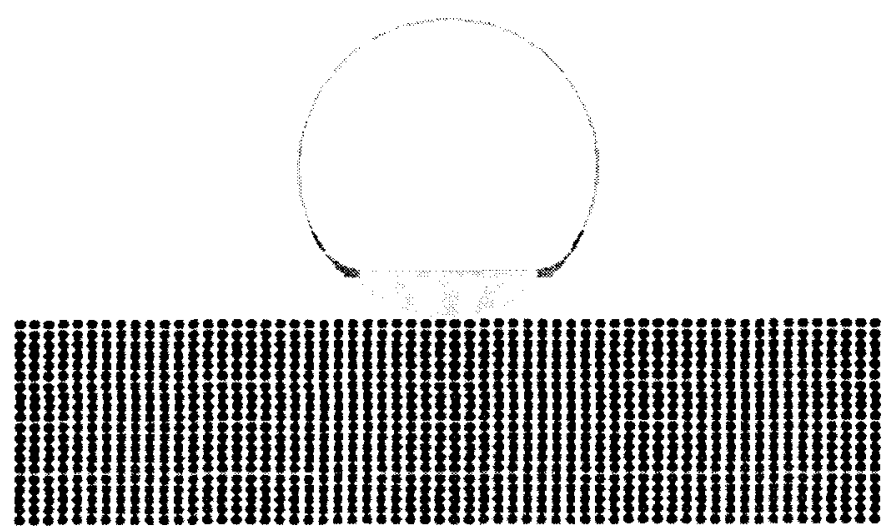

Figure 32. The section and water model using the SPH method. Note that no void is needed for this Lagrangian meshless method.

\section{Discussion of Results}

The crashworthy composite fuselage section was designed for a rigid surface impact. For a rigid surface impact, the bottom of each foam block begins to crush as soon as the contact pressure exceeds the crush stress. In the absence of friction, the contact force (and pressure) for a flat rigid surface is purely vertical. Consequently, the unsupported fiberglass skin between the blocks develops very low in-plane membrane forces. A "rigid" impact surface does not absorb any of the kinetic energy. However, for the water impact, very little crushing of the foam was detected. Thus, the water absorbed the major portion of the kinetic energy of the test article. Since water is an incompressible fluid, the collision involves momentum transfer from the fuselage to the water, which results in a large "splash." The unsupported fiberglass skin between the foam blocks receives the same initial pressure pulse as the supported skin. However, since the skin is unsupported from behind, it deforms inwardly to produce large in-plane membrane strains. Although the fiberglass subfloor skin did not fail for the rigid surface impact, multiple failures were observed in the subfloor skin after the water impact. Similar skin rupture has been observed in conventional metallic aircraft structures for water impacts. 
The finite element models were relatively insensitive to small variations in fluid density (as might occur from fresh to salt water). In addition, varying the water depth from $2.5-\mathrm{ft}$. to the actual $3.5-\mathrm{ft}$. pool depth did not produce a change in the initial acceleration pulse. Although LS-DYNA allows viscosity to be input into the equation of state, very little effect of viscosity was noticed on the filtered acceleration responses of the large masses on the floor. Both the MSC.Dytran and LS-DYNA models gave similar results for the 6- and 3-in. Euler meshes. The pulse shape and duration for the inboard and outboard floor locations were accurately predicted. The peak accelerations were higher than measured for both simulations and for both mesh refinements. However, in general, the finer 3-inch mesh showed better correlation.

\section{Acceleration, $\mathbf{g}$}

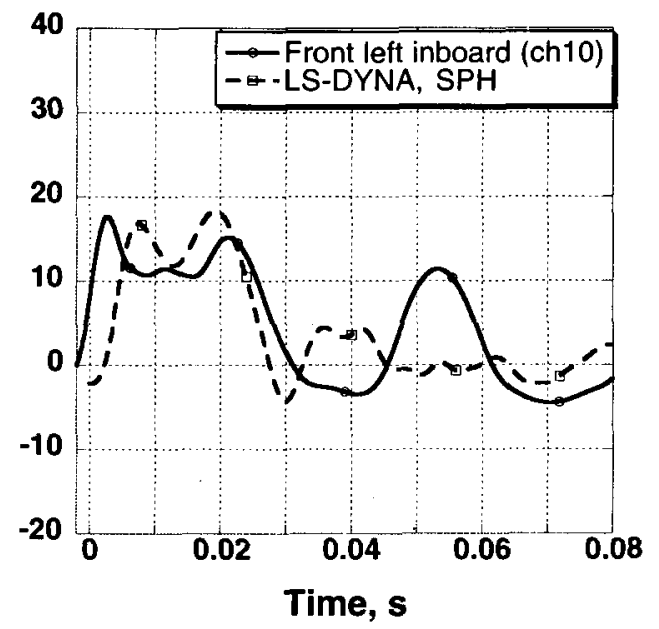

(a). Front left inboard, channel10.

\section{Acceleration, $\mathbf{g}$}

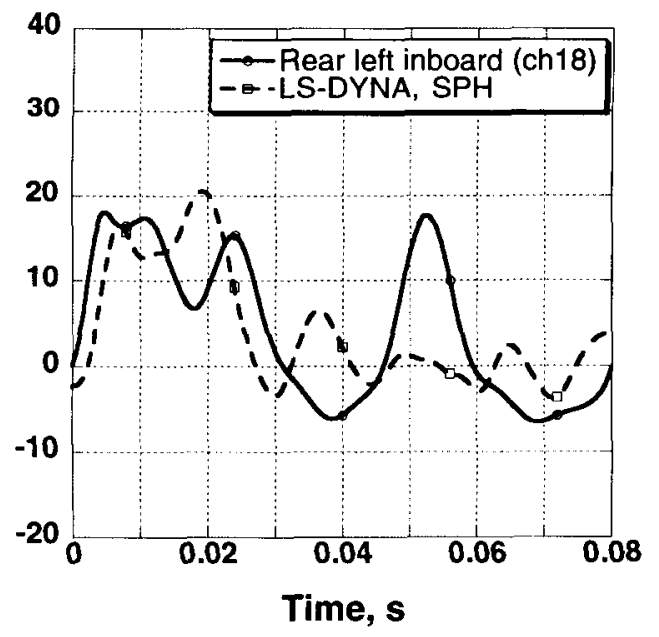

(b). Rear left inboard, channel 18.

Figure 33. Filtered experimental acceleration responses compared with LS-DYNA predicted inboard accelerations for the Lagrangian SPH solver.

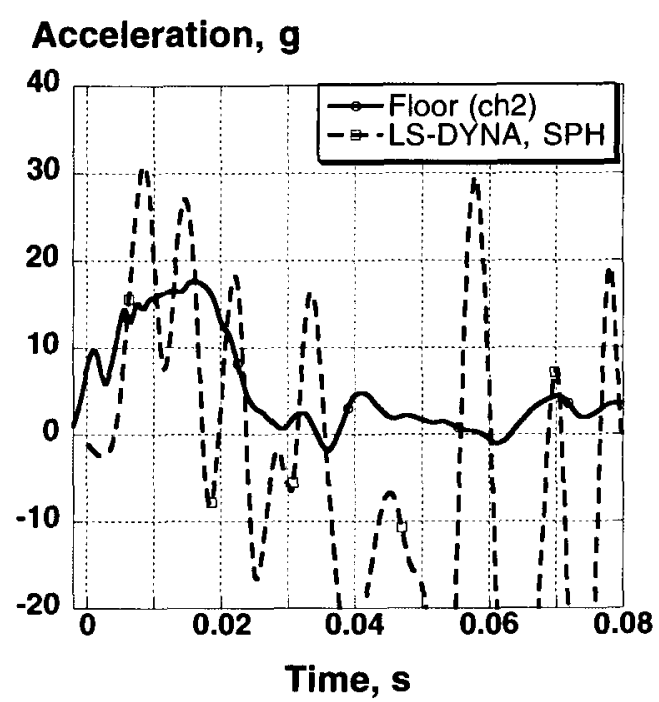

(a) Front left outboard floor-level, channel 2.

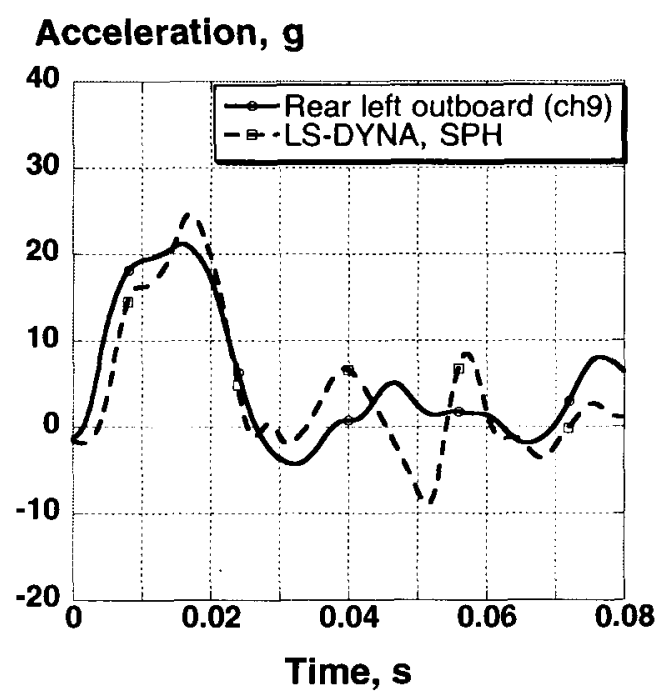

(b) Rear left outboard, channel 9.

Figure 34. Filtered experimental acceleration responses compared with LS-DYNA predicted outboard accelerations for the Lagrangian SPH solver.

The LS-DYNA model with the cylindrical tank correlated better with experimental data than the 3-inch rectangular tank model. The better results for the cylindrical tank model may be due to the slightly smaller Euler elements beneath the fuselage, and not the actual cylindrical shape of the Euler region. The 1inch gradient mesh and the SPH method gave the best results. Consequently, it appears that to obtain good results, the Euler mesh should be made sufficiently fine near the contact region. However, the mesh refinement has practical limits. For nonlinear problems, the solution may diverge if the mesh is made too fine. With the 1-inch gradient mesh, the Euler mesh had the same fineness as the bottom of the fuselage section. Thus, when failure was introduced into the bottom skin, water flowed through the failed regions of skin. Water did not flow through the failed skin for the 6-inch Euler mesh model. The 
introduction of a 2 percent failure strain to the bottom fuselage skin allowed water to penetrate though the failed region between the foam blocks. However, the initial peak acceleration was only reduced by a small amount from the original model without failure. More damage was predicted by the simulation with 2 percent failure strain than was observed experimentally. Consequently, the actual stress-strain curve to failure of the bottom skin laminate would be needed for an accurate simulation. Unfortunately, this information was not available.

\section{Concluding Remarks}

In March 2002, a 25-ft/s vertical drop test was conducted for a composite fuselage section impacting a $15-\mathrm{ft}$. diameter pool filled with water. The purpose of the test was to obtain experimental data for comparison with two previous drop tests that were performed onto a rigid surface and soft soil. For the drop test, the fuselage section was configured with ten 100 -lb. lead masses, five per side, that were attached to seat rails mounted to the floor. Test data were obtained from 67 accelerometers attached to the lead masses and 7 pressure transducers located within the foam blocks of the subfloor. Post-test examinations of the subfloor region indicated considerable damage to the outer skin for the water impact test. However, no subfloor crushing was observed. Some minor debonding of the face sheets from the foam core and fracturing of the outer "fingers" of the foam blocks were observed. No damage to the floor or upper fuselage cabin was evident. The floor-level acceleration responses were typically $20-\mathrm{g}$ in magnitude and approximately 0.03 seconds in duration.

Nonlinear dynamic finite element models using both Eulerian and Lagrangian formulations were constructed with the codes MSC.Dytran and LS-DYNA. For the MSC.Dytran simulations, the "fast" general coupling technique was used to simulate the fluid-structure interaction. MSC.Dytran simulations were executed for two different Euler mesh densities, one created with 6-in. cubic elements and one with 3 -in. cubic elements filling the same rectangular volume. The analytical predictions from the 3-in. Euler model were closer in magnitude and provided a better match to the overall shape of the experimental acceleration responses than did the results from the 6-in. Euler model. In addition, two water depths were modeled in MSC.Dytran. The shallow case was 5 cubes deep or 2.5 feet, while the larger depth was 7 cubes deep or 3.5 feet, the actual depth of the pool. No significant difference in the acceleration pulse for the first 0.03 seconds could be detected.

A series of LS-DYNA models were constructed to study the effects of the Euler mesh geometry and discretization, viscosity effects, and failure of the fuselage skin. The LSDYNA fuselage model was developed from the original MSC.Dytran model. ALE coupling was used to simulate the fluid structure interaction. The effect of water viscosity on the acceleration pulse was minimal. To assess the effect of mesh density and geometry, simulations were executed with both uniform 6-in. and 3-in. cubic Euler mesh discretizations. In addition, a cylindrical Euler mesh was constructed representative of the pool geometry. As compared with the experiment, the LS-DYNA models overpredicted the floor accelerations for the 6- and 3-in. Euler meshes. However, in general, the 3 -in. mesh gave better results. The LS-DYNA model with the cylindrical tank had smaller elements than the 3 -in. rectangular tank and gave improved correlation with test data. A final Euler model was constructed with a gradient mesh with the smallest 1 -in. elements located in close proximity to the bottom of the fuselage section. When failure strains were applied to this model, water did flow though the areas formed by the deleted failed elements. However, failure of the outer skin on the bottom of the fuselage section did not appreciably lower the initial peak accelerations. A final LSDYNA simulation was executed in which the water was modeled as Lagrangian using the SPH method. The predicted acceleration responses generated from the 1-in. gradient mesh and the SPH model showed the best agreement with the experimental results.

\section{References}

1. Sareen, A. K., Sparks, C., Mullins, B. R., Fasanella, E. L., and Jackson, K. E., "Comparison of Soft Soil and Hard Surface Impact Performance of a Crashworthy Composite Fuselage Concept," Proceedings of the AHS Forum 58, Montreal, Canada, June 11-13, 2002.

2. Pentecote, N. and Kindervater, C. M., Airframe Water Impact Analysis Using a Local/Global Methology, Proceedings of the AHS Forum 58, Montreal, Canada, June 1113, 2002.

3. Wittlin, G., Smith, M., Sareen, A., and Richard, M., Airframe Water Impact Analysis Using a Combined MSC/DYTRAN - DRI/KRASH Approach, Proceedings of the AHS Forum 53, Virginia Beach, VA, April 29 - May 2, 1997.

4. Lyle, K. H., Bark, L. W., Jackson, K. E., "Evaluation of Test/Analysis Correlation Methods for Crash Applications," Proceedings of the $57^{\text {th }}$ American Helicopter Society Annual Forum, Washington D.C., May 9-11, 2001.

5. Jackson, K. E., "Analytical Crash Simulation of Three Composite Fuselage Concepts and Experimental Correlation," Journal of the American Helicopter Society, Vol. 42, No. 2, April 1997, pp. 116-125.

6. Jackson, K. E., and Fasanella, E. L., "Innovative Composite Fuselage Design for Improved Crashworthiness," Proceedings of the 54th American Helicopter Society Forum and Technology Display, Washington DC, May 20-22, 1998.

7. Fasanella, E. L., and Jackson, K. E., "Analytical and Experimental Evaluation of Composite Energy Absorbing 
Subfloor Concepts," Proceedings of the AHS National Technical Specialists' Meeting on Rotorcraft Crashworthiness, Phoenix, AZ, Sept. 1998.

8. Jackson, K. E., and Fasanella, E. L., "Crashworthy Evaluation of a 1/5 Scale Model Composite Fuselage Concept," Proceedings of the 55th American Helicopter Society (AHS) Forum, Montreal, Canada, May 25-27, 1999.

9. Jackson, K. E., Fasanella, E. L., Kellas, S., "Development of a 1/5-Scale Model Composite Fuselage Section for Improved Crashworthiness," Journal of Aircraft, Vol. 38, No. 1, January-February, 2001, pp. 95103.

10. Jackson, K. E.; Kellas, S.; Fasanella, E. L., "Impact Testing and Simulation of a Crashworthy Composite Fuselage," Proceedings of the American Helicopter Society Forum 56, Virginia Beach, VA, May 2-4, 2000.

11. Jackson, K. E., and Fasanella, E. L., "Development of a Crashworthy Composite Fuselage Concept," Proceedings of the 2000 Advances in Aviation Safety Conference, Society of Automotive Engineering, P-355, April 2000, pp.37-50.

12. Jackson, K. E., Fasanella, E. L., Knight, N. F., "Demonstration of a Crashworthy Composite Fuselage Concept," Proceedings of the $22^{\text {nd }}$ Army Science Conference, Baltimore, Maryland, December 11-13, 2000.

13. Fasanella, E. L., Jackson, K. E., "Impact Testing and Simulation of a Crashworthy Composite Fuselage Section with Energy Absorbing Seats and Dummies," Proceedings of the AHS Forum 58, Montreal, Canada, June 11-13, 2002 .

14. Society of Automotive Engineers, Recommended Practice: Instrumentation for Impact Test - Part 1, Electronic Instrumentation, SAE J211/1, March 1995.

15. von Karman, T., The Impact of Seaplane Floats During Landing, NACA TN 321, Washington, D.C., Oct. 1929.

16. Anon., LS-DYNA Keyword User s Manual Volume I and II - Version 960, Livermore Software Technology Company, Livermore, CA, March 2001.

17. MSC.Dytran User s Manual Version 4.0, The MacNeal-Schwendler Corporation, Los Angeles, CA, November 1997.

18. Vignjevic, R. and Meo, M., Simulation of helicopter under-floor structure impact on water. International Journal of Crashworthiness, Vol. 6, No. 3., p 425-443.
19. Schmidt, R.M., Voss, M.E., Kari, S.E., Shrader, J.E.,"Explosive Loading of Lightweight Aircraft Structures",ASME/JSME Pressure Vessels and Piping Conference, Honolulu, Hawaii, July 23-27, 1995, Symposium on Structures under Extreme Loading Conditions, ASME PVPVol. 299, P107-116. 